\title{
Sensory cortical dynamics during optical microstimulation training
}

Pancholi, Ravi; Ryan, Lauren; Peron, Simon

Center for Neural Science, New York University, 4 Washington PI., Rm. 621, New York, NY 10003

Lead Contact: Simon Peron, speron@nyu.edu

\section{SUMMARY}

Primary sensory cortex is a key locus of plasticity during learning. Exposure to novel stimuli often alters cortical activity, but isolating cortex-specific dynamics is challenging due to extensive pre-cortical processing. Here, we employ optical microstimulation of pyramidal neurons in layer (L) 2/3 of mouse primary vibrissal somatosensory cortex (vS1) to study cortical dynamics as mice learn to discriminate microstimulation intensity. Tracking activity over weeks using two-photon calcium imaging, we observe a rapid sparsification of the photoresponsive population, with the most responsive neurons exhibiting the largest declines in responsiveness. Following sparsification, the photoresponsive population attains a stable rate of neuronal turnover. At the same time, the photoresponsive population increasingly overlaps with populations encoding whisker movement and touch. Finally, we find that mice with larger declines in responsiveness learn the task more slowly than mice with smaller declines. Our results reveal that microstimulation-evoked cortical activity undergoes extensive reorganization during task learning and that the dynamics of this reorganization impact perception. 


\section{INTRODUCTION}

A defining characteristic of sensory cortex is its ability to change with experience and use (Feldman, 2009). A major driver of this plasticity is an animal's frequent exposure to novel sensory input. The brain must encode these novel stimuli and use them to inform behavior. Sensory cortical activity undergoes a range of changes in response to novel, reward-predictive stimuli. In some cases, individual neurons show an increased preference for task-relevant stimuli (Poort et al., 2015; Rutkowski and Weinberger, 2005), and populations selective for these stimuli increase in size (Chen et al., 2015). In other cases, sensory representations in cortex remain stable in size and population-scale tuning (Peron et al., 2015). However, even when representations are stable at the population scale, high levels of turnover are observed in individual neurons (Deitch et al., 2021; Marks and Goard, 2021; Perez-Ortega et al., 2021; Peron et al., 2015; Schoonover et al., 2021). Understanding representational stability is crucial as it imposes important constraints on downstream readout and thereby limits perception (Clopath et al., 2017).

Whether repeated exposure to novel input will increase or reduce sensory cortical activity remains unclear. Theoretical work demonstrates that repeated activation of a group of pyramidal neurons can result in both increases and reductions in responsiveness (Sadeh and Clopath, 2021). In models, repeated activation by a novel input pattern can lead to the emergence of densely interconnected, input-selective subnetworks (Clopath et al., 2010; Litwin-Kumar and Doiron, 2014), which produce increased responses to stimulation due to recurrent amplification (Peron et al., 2020). However, such models depend on mechanisms such as increased inhibition to prevent runaway synaptic potentiation (Litwin-Kumar and Doiron, 2014; Vogels et al., 2011), which may result in reduced responsiveness overall. Experimental evidence shows that both increased and reduced responsiveness can result from repeated stimulation of vibrissal primary somatosensory cortex (vS1). For example, co-activation of subsets of excitatory neurons in mouse vS1 results in elevated excitability among the stimulated population, potentially due to increased connectivity (Kim et al., 2016). At the same time, repeated activation of vS1 via whisker stimulation leads to elevated inhibitory input onto cortical pyramidal neurons (Knott et al., 2002). However, because most learning experiments employ naturalistic stimuli, pre-cortical sources of plasticity can contribute to the observed dynamics (Audette et al., 2019; Wimmer et al., 2010). Moreover, naturalistic sensory input independently activates multiple cortical areas, so that the perceptual contribution of any given area is limited. Thus, whether sensory cortical activity increases or declines in response to novel, behaviorally relevant input and how this impacts perception remains unclear.

Cortical microstimulation has been used extensively in the study of perception as it allows for direct control of cortical activity (Histed et al., 2012). Electrical microstimulation of cortex can drive perceptual report (Afraz et al., 2006; Penfield and Boldery, 1937; Salzman et al., 1990; Yang and Zador, 2012), even upon stimulation of single neurons (Houweling and Brecht, 2008; Tanke et al., 2018). However, electrical microstimulation activates a distributed population of neurons that is poorly confined in space (Histed et al., 2009), is difficult to pair with recording due to a pronounced stimulus artifact (Weiss et al., 2019), and can cause gliosis and tissue pathology if placed chronically (Polikov et 
al., 2005). Optical microstimulation is an alternative to electrical microstimulation that avoids these problems and further allows for refined spatial and genetic control of the evoked activity. In mouse vS1, optical microstimulation can reliably drive behavioral report following the activation of a small number of neurons (Dalgleish et al., 2020; Huber et al., 2008; O'Connor et al., 2013). This makes optical microstimulation especially well-suited for probing cortical dynamics during learning.

Here, we train mice to report the intensity of optical microstimulation-evoked activity in a subset of vS1 layer (L) 2/3 pyramidal neurons. Over the course of training, we track neural dynamics in the opsin-expressing volume using two-photon calcium imaging. We observe a rapid reduction in responsiveness among both opsin-expressing and opsin nonexpressing neurons, with the most responsive neurons exhibiting the largest declines in responsiveness. Following sparsification, a steady state is attained in which the number of neurons that cease responding to photostimulation matches the number of neurons that become photoresponsive on any given day. In addition, the photoresponsive population comes to increasingly overlap with vS1 populations encoding whisker movement and touch. Finally, we find that animals that exhibit greater sparsification are less likely to learn the task than animals with less sparsification. Together, these results show that primary sensory cortex exhibits complex dynamics in response to a novel, behaviorally relevant stimulus and that these dynamics impact perception. 


\section{RESULTS}

\section{Training mice to perform an optical microstimulation discrimination task}

We studied cortical dynamics as mice were trained on an optical microstimulation task. Transgenic mice expressing GCaMP6s in cortical excitatory neurons (Slc17a7-Cre X Ai162) (Daigle et al., 2018) were virally injected with the soma-restricted opsin ChRmine (AAV-8-CaMKIla-ChRmine-mScarlet-Kv2.1-WPRE) (Marshel et al., 2019) in layer (L) 2/3 of primary vibrissal somatosensory cortex (vS1). To allow optical access, a cranial window was implanted over vS1. Following recovery, we used widefield imaging during whisker deflection to confirm that opsin expression was restricted to vS1 (typically the C row of whiskers; Figure S1). A miniature light-emitting diode (LED) was affixed to the cranial window adjacent to the site of opsin expression for optogenetic stimulation (Figure 1A).

LED pulses ( $5 \mathrm{~ms}$, minimal interval: $50 \mathrm{~ms}$ ) were delivered to the opsin-expressing area and animals required to report whether the number of photostimulation pulses was high or low. Mice were rewarded for licking the left of two lickports on low pulse count trials (1 or 3 pulses; 0 pulses in early training) and the right lickport on high pulse counts (7 or 9 pulses), with 5 pulse count trials rewarded randomly (Figure 1B). LED output was stable within and across training sessions (Figure S2). We found that increasing the pulse count, the pulse amplitude, or the inter-pulse interval all resulted in greater photostimulation-evoked activity (Histed and Maunsell, 2014) (Figure S3). On each trial, photostimulus pulses were presented during the stimulus epoch $(500 \mathrm{~ms})$. This was followed by a short delay period $(500 \mathrm{~ms})$, after which mice could indicate their response by licking during the response epoch ( $<2 \mathrm{~s})$ (Figure 1C). Stimulus pulses were randomly permuted among 9 time bins to prevent animals from using timing cues to solve the task (Figures 1D and S4). A spectrally matched LED was placed near the mouse and illuminated during each time bin to prevent animals from using visual cues to solve the task (Figure 1B).

Mice were first trained on a photostimulation detection task (9 pulses vs. 0 pulses, 2 lickports; Figure 1E). Mice reached criterion performance (d-prime $>1.5$ ) on this task in $9.8 \pm 3.7$ days (mean $\pm S D ; n=6$ mice), after which additional pulse counts were added until mice were proficient at the full pulse count discrimination task. Mice achieved high performance at this final stage $(78.3 \% \pm 3.4 \%$ correct, $n=6$ mice; Figures $\mathbf{1 F}$ and $1 \mathbf{G})$. To confirm that animals required opsin expression to perform the task, we trained a separate cohort of mice not infected with the opsin (Figure 1F). These mice could not learn the task after 20 training sessions (performance on final day: $48.6 \% \pm 1.4 \%$ correct, $\mathrm{n}=5$ mice). Moreover, lesioning the opsin-expressing area in mice that had progressed to the discrimination task degraded task performance (Figure $\mathbf{1 H}$; average performance of 3 sessions before lesion: $78.8 \%$ correct, 3 sessions after lesion: $60.9 \%$, p-value $=$ 0.014 , paired t-test). Thus, mice can learn to discriminate the number of photostimulation pulses delivered to a subset of neurons in L2/3 of vS1 via optical microstimulation.

\section{Optical microstimulation evokes reliable responses}


To assess neural activity during optical microstimulation, we recorded from L2/3 of the opsin-expressing area using volumetric two-photon calcium imaging. Neurons distributed among three 800-by-800 $\mu \mathrm{m}$ imaging planes spaced $60 \mu \mathrm{m}$ apart were tracked for 29.8 \pm 6.9 days (mean $\pm \mathrm{SD} ; \mathrm{n}=6$ mice; Figure $\mathbf{2 A}$ ). Imaged neurons were separated into opsin-expressing (692.6 \pm 123.2 neurons per mouse) and opsin non-expressing (2287.3 \pm 466.9 neurons per mouse) populations based on the presence of mScarlet (Methods). Based on past estimates of L2/3 pyramidal neuron density (Lefort et al., 2009; Peron et al., 2015), we imaged $15-20 \%$ of infected $L 2 / 3$ neurons, for a total of $\sim 3,500$ to $\sim 4,500$ opsin-expressing neurons per mouse. Neurons exhibited diverse responses to photostimulation (Figures 2B and 2C). We identified both opsin-expressing and opsin non-expressing excitatory neurons that responded to photostimulation, with photoresponsive neurons showing monotonically increasing response amplitudes (measured using z-scored $\Delta \mathrm{F} / \mathrm{F}$; Methods) with increasing pulse count (Figure 2D). Photoresponsive neurons were broadly distributed and intermingled (Figure 2E). In welltrained mice, higher photostimulus pulse count consistently increased the fraction of neurons responsive to stimulation (Figure 2F), as well as the response amplitude (Figure $2 \mathrm{G}$ ) and response probability (Figure $\mathbf{2 H}$ ) of individual neurons. This was true for both opsin-expressing and opsin non-expressing neurons. In all cases, the opsin-expressing population responded more reliably and strongly to photostimulation than the opsin nonexpressing population.

Electrical (Matyas et al., 2010) and optical (Auffret et al., 2018) stimulation of vS1 produces retraction of the contralateral whisker, presumably via activation of $L 5$ pyramidal neurons. We employed high-speed videography to capture contralateral whisker movement during our task and determine whether our stimulus directly drove whisking. On the first day of training, we did not observe any difference in whisker movement between photostimulated and non-photostimulated trials (Figure S5). Over time, however, we found that stereotypical whisking patterns emerged. Thus, while animals learn to whisk during the stimulus epoch and some interact with the photostimulus, the diversity of whisking patterns across animals and lack of evoked retractions on the first few days of training implies that photostimulation does not directly drive whisker movement.

\section{Activity rapidly sparsens during optical microstimulation task training}

Sensory cortical representations in L2/3 exhibit diverse dynamics during learning (Peron et al., 2015; Poort et al., 2015). By tracking neural activity as mice learned the optical microstimulation task, we were able to assess how cortex responds to directly evoked, behaviorally relevant activity (Figures 3A and 3B). Restricting our analysis to trials with 9 photostimulus pulses, which were present in all sessions, we identified distinct neurons with stable, increasing, or decreasing responses to photostimulation (Figure 3C). These dynamics were present in both the opsin-expressing and opsin non-expressing populations. We found that the most responsive neurons exhibited the largest declines in response amplitude and probability across training (Figures 3D and 3E). Though responsiveness did not change for the majority of the recorded population, we observed a significant decline in response amplitude and probability for those neurons whose 
responses exceeded the $95^{\text {th }}$ or $99^{\text {th }}$ percentiles of response amplitudes on at least one day (Methods) (Figure 3F). Thus, optical microstimulation task training results in a sparsification of responses to photostimulation, primarily due to declining responses among the most responsive neurons.

To characterize the response dynamics of neurons active at different phases of training, we next asked how neurons that were highly responsive (top $5 \%$ by response amplitude; Methods) during early, middle, or late sessions changed over time (Figure 3G). The fraction of highly responsive opsin-expressing neurons declined over early training, from $0.29 \pm 0.04$ on early sessions to $0.16 \pm 0.05$ on middle sessions $(p<0.001$, paired t-test; Figure $3 \mathrm{H})$, reaching $0.11 \pm 0.05$ on late sessions $(p=0.084$, middle vs. late). The fraction of highly responsive opsin non-expressing neurons remained unchanged, going from 0.08 \pm 0.05 on early sessions to $0.04 \pm 0.01$ on middle sessions ( $p=0.280$, paired t-test), and reaching $0.03 \pm 0.01$ on late sessions ( $p=0.099$, middle vs. late). Examining each population in isolation, we find that highly responsive opsin-expressing and opsin nonexpressing neurons from early sessions exhibit a large decline in response amplitude and probability across training, with the largest decline in responsiveness between early and middle sessions (Figures $\mathbf{3} \mathbf{I}$ and $\mathbf{3} \mathbf{J}$ ). In contrast, the most responsive neurons in middle sessions show only a modest decline in responsiveness between middle and late sessions. We also find that highly responsive opsin non-expressing neurons from late sessions show a modest increase in response amplitude and probability between early and middle sessions. Thus, optical microstimulation training drives a host of changes within the stimulated population, with the most prominent being a decline in responsiveness among the top early responders.

To control for changes in responsiveness due to changes in opsin expression, we tracked mScarlet fluorescence ('redness') over the course of training (Figure S6A; Methods). We found that overall redness across all neurons did not change over the course of our experiment $(p=0.490$, paired t-test, $n=6$ mice; Figure S6B). To determine whether individual neurons' expression changed within the population, we computed the correlation between the vector of redness values across all neurons on one day with an equivalent vector on other days (Figure S6C). We found that the levels of redness across days remained consistent across the population (Figure S6D). Thus, observed changes in neural responsiveness were not due to changes in opsin expression.

We next asked whether opsin expression was a reliable predictor of responsiveness. On early training days, redness was correlated with response amplitude (Figure S7A). On middle and late sessions, however, this relationship disappeared (Figure S7B). Whereas the decline in this correlation was significant from early to middle sessions ( $p=0.046$, paired t-test comparing early vs. middle, $\mathrm{n}=6$ mice), it was not significant from middle to late sessions $(p=1)$. Given that highly responsive neurons showed the largest declines in response, we asked whether the neurons with the largest response declines also the had the highest levels of opsin expression. We assessed this by examining the relationship between redness and change in response amplitude from early to late sessions. We found that in individual animals, the neurons with the highest levels of opsin expression exhibited the largest declines in responsiveness (Figure S7C). Dividing opsin- 
expressing neurons into three equal sized bins based on their relative redness, we found that only the neurons showing the highest levels of mScarlet fluorescence exhibited significant declines in responsiveness from early to late training (Figure S7D). One possible basis for this decline is reduced excitability. To quantify excitability over time, we measured the spontaneous calcium event rate during non-stimulus epochs. We found no relationship between redness and changes in spontaneous event rate (Figure S7E). Thus, the neurons with the highest levels of opsin expression exhibit the largest declines in responsiveness from early to late sessions, and opsin expression only predicts responsiveness in early training.

\section{Representational drift reaches a steady state following sparsification}

Representational drift-the gradual change in the responsiveness and identity of neurons responding to a particular stimulus - has been observed in many cortical areas (Rule et al., 2019). Is such drift intrinsic to cortex? To examine the stability of the responsive population at the individual neuron level, we first examined how the relative responsiveness of neurons changed across days. For each day, we constructed a vector consisting of the mean response amplitude to 9-pulse stimuli across all photoresponsive neurons on at least one day. We then computed the Pearson correlation between these vectors across all pairs of days (Figure 4A). This correlation declined over longer timescales, implying that the responses of neurons to photostimulation changed over time (Figure 4B). Over the course of training, however, the rate of change slowed. We quantified this by examining the number of days until $\mathrm{R}^{2}$ for the mean response amplitude across days dropped below 0.5 (Figure 4C). For opsin-expressing neurons, this grew from $1.9 \pm 0.9$ days in early training (days $1-3$ ) to $6.4 \pm 3.1$ in later training (days $9-11 ; p$ $=0.009$, paired t-test, $\mathrm{n}=6$ mice). Opsin non-expressing neurons showed no change (early, $3.6 \pm 2.3$ days; late, $3.9 \pm 2.1$ days; $p=0.869$ ). Thus, for opsin-expressing but not opsin non-expressing neurons, the rate of change in responsiveness slowed in late training. However, a steady rate of change was still observed for all neurons at this later stage.

We next examined the stability of representation membership by examining streaks of consecutive sessions in which a neuron was responsive. We tracked response probability across days (Figure 4D) for highly responsive neurons ( $P$ (response) $>0.5$ for at least one day), treating these neurons as responsive on a given day if their response probability exceeded 0.1 for that day (Figure 4E). We first asked how many streaks each neuron participated in. The majority $(79.4 \% \pm 10.0 \% ; n=6$ mice $)$ of responsive opsin-expressing neurons exhibited a single streak (Figure 4F), as did most opsin non-expressing neurons $(69.9 \% \pm 8.8 \%)$. We next examined the length of streaks, finding that the most common length was one day, though about two-thirds of streaks were longer for both opsinexpressing and opsin non-expressing populations (Figure 4G).

Because our approach captured streak onset and offset on any given training day, we could measure the rate with which new neurons entered the responsive population as well as the rate with which existing members departed. The onset rate matched the offset rate for opsin non-expressing neurons across all time points, and reached a similar state for opsin-expressing neurons after around two weeks (Figure $\mathbf{4 H}$ ). With respect to opsin- 
expressing neurons, in the latest epoch examined (days 20-22), $0.5 \pm 0.3 \%$ entered the responsive representation whereas $0.7 \pm 0.2 \%$ departed. For opsin non-expressing neurons, these numbers were $0.2 \pm 0.2 \%$ and $0.2 \pm 0.2 \%$, respectively. Thus, assuming no replacement, it would take approximately 0.5-2 years for all neurons in the imaged area to have participated in the responsive population.

The responsive population, however, is a subset of the overall population and thus turns over more rapidly. Of the photoresponsive opsin-expressing neurons, $3.6 \pm 0.9 \%$ became non-responsive on any given day, with $2.8 \pm 1.3 \%$ new neurons being added daily. Of the photoresponsive opsin non-expressing neurons, $3.6 \pm 0.8 \%$ became non-responsive whereas $3.6 \pm 1.4 \%$ were added. Assuming no replacement, this implies a complete turnover of the responsive population within approximately one month. Given the stable, high performance of expert mice in our task (Figures 1E and 1F), downstream readout of photostimulation is capable of adapting to steady turnover among the photoresponsive population.

In sum, even though the size of the photoresponsive population and amount of evoked activity stabilize after a few days of training, the neurons that make up this stable group continue to change with time.

The photoresponsive population overlaps increasingly with vibrissal-responsive populations

Vibrissal S1 contains neurons that encode vibrissal movement ('whisking') and vibrissal touch. Both representations are stable when mice learn a vibrissal object localization task (Peron et al., 2015). How does the relationship between the photoresponsive population and the vibrissal populations change during optical microstimulation task training? To address this, we trimmed mice to contralateral whiskers $\mathrm{C} 1-3$ and tracked these whiskers across all imaging sessions (Figure 5A). Furthermore, in a task-free session, we mapped vibrissal touch responses for the spared vibrissae by repeatedly pressing a pole against each of the whiskers individually while imaging (Figure 5B; Methods).

We used an encoding model (Peron et al., 2015) to classify touch and whisking neurons (Methods). This encoding model predicts neural activity from vibrissal kinematics, yielding a touch and whisking score for each neuron in the form of the Pearson correlation between the model-predicted $\Delta F / F$ and the neural $\Delta F / F$. The representation of whisking was stable over the course of optogenetic task training, though individual neurons did exhibit turnover (Figure S8). We developed a second encoding model to predict neural activity from the optogenetic stimulus, yielding an analogous Pearson correlation-based encoding score for photostimulation (Figure S9; Methods). Using encoding model scores, we assigned neurons to the photoresponsive, whisking, and touch populations, allowing us to quantify the overlap between these representations. The photoresponsive population exhibited overlap with both the touch and whisking populations (Figure 5C). In addition, the touch populations for adjacent whiskers also exhibited overlap.

Over the course of training, the photoresponsive population came to increasingly overlap with both the touch and whisking populations (Figure 5D). To quantify this overlap, we computed the overlap between pairs of populations, using the size of intersection divided 
by the size of union (Jaccard similarity; Methods). We further computed a shuffled overlap by randomly drawing two groups of neurons from either the opsin-expressing or opsin non-expressing neurons and determining their overlap. By dividing the actual overlap by this shuffled overlap, we obtained a normalized fractional overlap, with values significantly above one implying greater-than-chance overlap and values significantly below one indicating below-chance overlap. For opsin non-expressing neurons, the normalized overlap between the photoresponsive and touch populations was significantly greater than one for early (Figure 5E; normalized overlap: $1.39 \pm 0.09 ; p=0.024$, t-test testing for difference from a distribution having a mean of 1 and identical variance, $n=6$ mice), middle $(1.69 \pm 0.12 ; p=0.009)$, and late sessions $(1.99 \pm 0.21 ; p=0.015)$. This was also true of the overlap with the whisking population on early (normalized overlap: $1.64 \pm 0.08$; $p=0.024)$, middle $(2.03 \pm 0.20 ; p=0.030)$, and late sessions $(3.00 \pm 0.43 ; p=0.024)$. For opsin-expressing neurons, there was no significant overlap between the photoresponsive and touch populations in any phase of training (Figure 5F; early: $1.03 \pm$ $0.05 ; p=1$; mid: $1.27 \pm 0.09, p=0.093$; late: $1.43 \pm 0.19, p=0.207$ ), and the photoresponsive population only significantly overlapped with the whisking population in middle sessions (early normalized overlap: $1.24 \pm 0.06$; $p=0.126$; mid: $1.60 \pm 0.12, p=$ 0.030 ; late: $1.51 \pm 0.20, p=0.660$ ). In all cases, the overlap among opsin non-expressing neurons was greater than for opsin-expressing neurons. As a control, we examined overlap between pairs of touch populations for a single day, which always exceeded chance (opsin non-expressing: $2.15 \pm 0.22, p=0.003$; opsin-expressing: $2.15 \pm 0.36, p=$ 0.024 ), as expected from the preponderance of multi-whisker responsive neurons in L2/3 of vS1 (Clancy et al., 2015).

Thus, the photoresponsive population comes to increasingly overlap with both the touch and whisking populations. This effect is mostly confined to opsin non-expressing neurons, which show a greater-than-chance overlap with vibrissal populations from training onset. This suggests that neural activity is channeled towards specific response patterns by the structure of local microcircuitry.

\section{Representational stability predicts the speed of learning}

Can differences in neural dynamics during microstimulation task training impact the rate of learning? To address this question, we analyzed photostimulus responses in several additional mice that were imaged only briefly due to their slow learning rate ('nonlearners'). We asked whether the fraction of highly photoresponsive neurons at training onset (neurons in the first training session exceeding the $95^{\text {th }}$ percentile of responsiveness across all sessions; Methods) could predict learning. We found no measurable difference in this fraction between animals that learned the task and those that did not (Figure 6A; $p=0.888$, t-test comparing $n=6$ learners with $n=4$ non-learners). Furthermore, the correlation between the fraction of highly photoresponsive neurons in the first training session and performance on the final common day of training (day 9) across all mice was not significant (Figure 6B; $p=0.360, n=10$ mice). Thus, the level of evoked activity on the first day of training does not predict the rate of learning.

Given that responses to photostimulation declined at different rates in different animals (Figures $\mathbf{3 H}-\mathbf{J}$ ), we next asked whether the magnitude of the decline in responsiveness 
could predict learning. Greater response decline would imply a larger change in evoked activity. This may increase the difficulty of downstream decoding and decrease the likelihood of learning. Consistent with this, we found that the decline in the fraction of highly photoresponsive neurons from day 1 to day 9 is larger among non-learners than learners (Figure $\mathbf{6 C}, p=0.005$ ). In addition, this change in responsiveness could predict the performance on the final common day of training (Figure 6D, $R=0.71$, Pearson correlation, $p=0.022, n=10$ mice). Thus, mice that did not learn the task exhibited larger declines in responsiveness than those that learned within the same period of imaging. 


\section{DISCUSSION}

We track neural activity in vS1 over several weeks as mice learn to perform an optical microstimulation task. We find that the photoresponsive population initially sparsens, with the largest declines in responsiveness observed among the most responsive neurons. Following sparsification, the photoresponsive population remains dynamic and exhibits a steady rate of neuronal turnover. At the same time, the photoresponsive population comes to increasingly overlap with natural vibrissal sensory populations. Finally, learning is fastest in animals whose photoresponsive population is the most stable over the course of training. Our results demonstrate that direct sensory cortical stimulation drives cortical reorganization and that these dynamics impact perception.

Microstimulation has been used extensively in the study of perception (Cohen and Newsome, 2004; Histed et al., 2012), yet tracking the dynamics of microstimulationevoked activity over learning has proven difficult. Chronic electrical microstimulation poses numerous challenges: it is invasive, can inadvertently activate neurons up to millimeters away from the stimulation site (Histed et al., 2009), and can cause tissue pathology at the site of electrode insertion (Polikov et al., 2005). Furthermore, simultaneous recording is complicated by a prominent stimulation artifact (Weiss et al., 2019). Finally, it cannot target genetically separable neural populations. Optical microstimulation overcomes these issues. Photostimulation using an external LED does not require an intracortical implant, the stimulation is spatially and genetically confined, and calcium imaging allows us to observe evoked responses within thousands of neurons at single cell resolution (Dhawale et al., 2017). In addition, mice can readily learn to detect optical microstimulation of vS1 (Dalgleish et al., 2020; Finkelstein et al., 2021; Huber et al., 2008). In contrast to objective-based optogenetic illumination, our LED-based approach also allows the objective to move without impacting photostimulation. This allows for imaging at multiple depths and in other brain areas, as well as high throughput training away from the microscope.

We observe sparsification of the photoresponsive population over the course of the first week of training, both in terms of the size of the responsive population and the typical response for a given neuron. With novel natural stimuli, both increases and decreases in responsive populations have been observed. For example, novel, behaviorally-relevant tones become overrepresented across learning in auditory cortex, (Weinberger, 2004) whereas whisker representations shrink following environmental enrichment in vS1 (Polley et al., 2004). Candidate mechanisms for the latter decline in responsiveness include increased inhibitory input onto pyramidal neurons (Knott et al., 2002) and reduced excitability (Barth et al., 2004; Desai et al., 1999). Given that the most active neurons experience the largest response declines, sparsification is unlikely to be due to global mechanisms, such as a general increase in inhibition.

Direct optogenetic stimulation of small (Kim et al., 2016) and large (Zhang et al., 2020) cortical populations drives elevated responsiveness, potentially due to elevated connectivity among stimulated neurons. Our observation of declining responsiveness does not rule out increased connectivity among the remaining responsive neurons, as would be expected from plasticity rules governing excitatory-excitatory synapses within 
L2/3 of vS1 (Banerjee et al., 2014). Repeated photostimulation of groups of pyramidal neurons can drive elevated connectivity (Carrillo-Reid et al., 2016; Kim et al., 2016), though it can also cause enhanced excitability (Alejandre-García et al., 2020; Zhang et al., 2020). Determining whether chronic microstimulation drives changes in connectivity among photoresponsive pyramidal neurons will require further investigation.

Representational drift has been observed across primary sensory cortices (Clopath et al., 2017; Deitch et al., 2021; Marks and Goard, 2021; Perez-Ortega et al., 2021; Peron et al., 2015; Schoonover et al., 2021), though it is likely that pre-cortical structures contribute to drift when stimuli engage the entire sensory pathway. We observe representational drift in the context of direct cortical stimulation, implying that such turnover is an intrinsic feature of cortex. Moreover, our observation that learning is slowest in animals with the most rapidly changing photoresponsive population suggests that excessively dynamic representations are more difficult to use in associative learning.

Whether activating a random subset of an area's excitatory neurons should engage the same neurons as naturalistic stimuli is unclear. Evidence suggests that cortical activity is often confined to a small subspace of possible activity (Chung and Abbott, 2021). For instance, spontaneous activity resembles evoked activity in visual, auditory, and somatosensory cortices (Kenet et al., 2003; Luczak et al., 2009). In addition, direct activation of a subset of visual cortical neurons with particular tuning can drive activity in the remaining neurons with similar tuning (Carrillo-Reid et al., 2019; Marshel et al., 2019). At the same time, the dominant interaction among distinctly tuned visual cortical excitatory neurons is mutual suppression (Chettih and Harvey, 2019). We find that the opsin nonexpressing photoresponsive population comes to increasingly overlap with the whisker movement-responsive and touch-responsive populations. Given the existence of a small subset of L2/3 vS1 neurons that are disproportionately excitable (Crochet et al., 2011), it is possible that both natural and artificial excitation engages this subpopulation. Furthermore, given the recurrent amplification observed in vS1 touch neurons (Peron et al., 2020), the increasing overlap of the photoresponsive population with the touch population suggests that recurrent circuitry can act as an attractor for initially random activity.

Sensory cortical feedback is important for the design of effective neural prosthetics (Pandarinath and Bensmaia, 2021; Tyler, 2015). Such feedback can reproduce subtle features of somatosensory percepts, such as vibration frequency (Romo et al., 1998), and recapitulate tactile sensations that improve the control of prosthetic limbs (Flesher et al., 2021). Our work suggests somatosensory cortical activity will stabilize at different rates in different individuals, and that this impacts perception. The increasing overlap between evoked populations and vibrissal sensory populations suggests that under some circumstances, repeated cortical feedback may come to evoke touch-like activity patterns. This may account for the efficacy of substituting artificial somatosensory percepts for natural percepts (O'Connor et al., 2013; Romo et al., 1998), and the longstanding observation that cortical stimulation can evoke complex, natural sensations (Penfield and Boldery, 1937). 
bioRxiv preprint doi: https://doi.org/10.1101/2021.12.17.473191; this version posted December 20, 2021. The copyright holder for this preprint

(which was not certified by peer review) is the author/funder, who has granted bioRxiv a license to display the preprint in perpetuity. It is made available under aCC-BY-NC-ND 4.0 International license.

We show that over the course of optical microstimulation task training, activity in the stimulated region of vS1 undergoes extensive reorganization, dominated by reduced responsiveness among the most responsive neurons. We also find that animals with larger reductions in activity exhibit the slowest rates of task learning. Microstimulation thus evokes specific sensory cortical dynamics that have a direct impact on the speed of associative learning. 


\section{ACKNOWLEDGMENTS}

We thank Claudia Clopath, Sadrah Sadeh, Robert Froemke, Anthony Movshon, and Michael Long for discussion. We thank Bettina Voelcker, Keelin O'Neil, and Aaron Lanz for discussion and technical assistance. This work was supported by the Whitehall Foundation and the National Institutes of Health (R01NS117536; F31NS120483; T32GM007308).

\section{AUTHOR CONTRIBUTIONS}

R.P. and S.P. designed the study. R.P. carried out all the experiments. L.R. performed histology and developed the lesion method. R.P. and S.P. performed data analysis and wrote the paper.

\section{DECLARATION OF INTERESTS}

The authors declare no competing interests. 
Pancholi et al., Figure 1

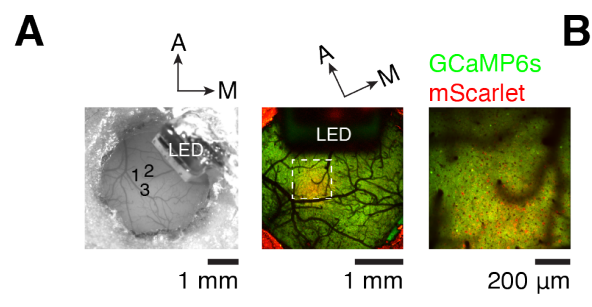

E
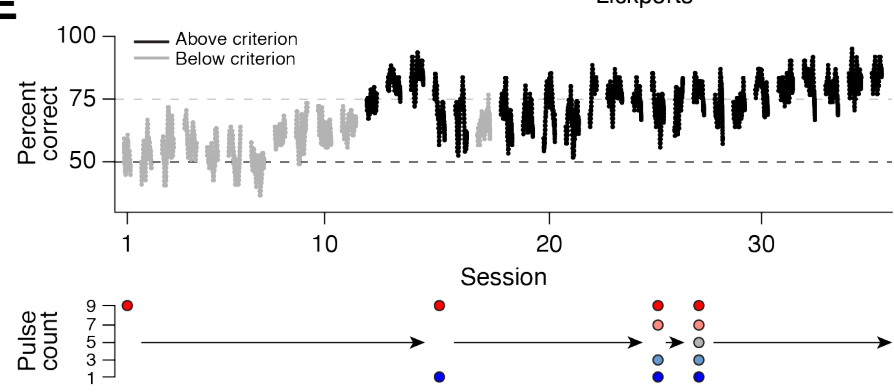

$\mathbf{F}$

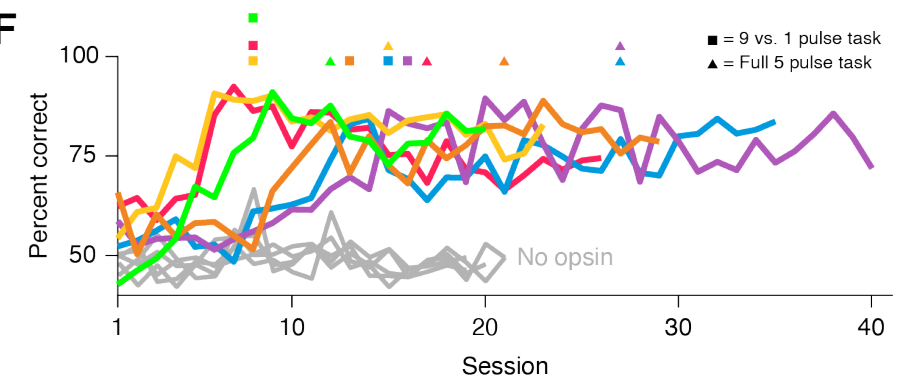

C

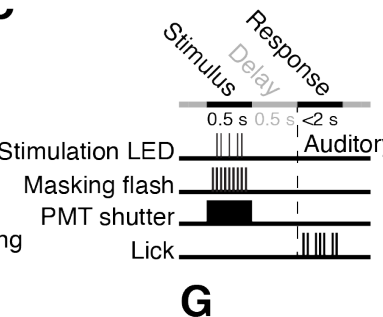

D
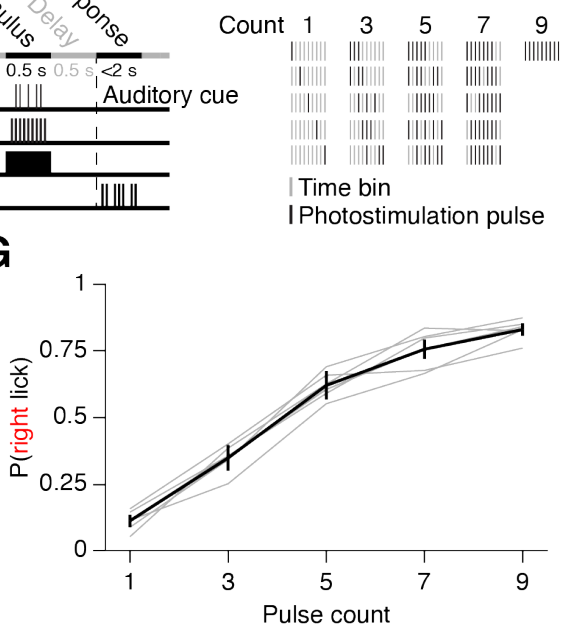

H
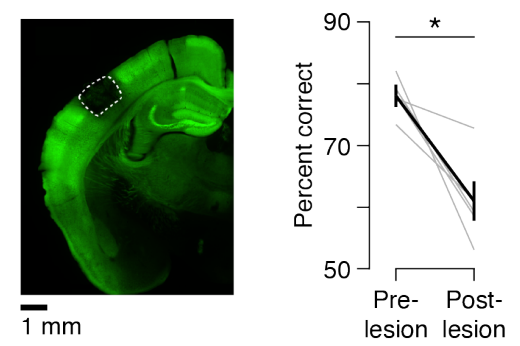

Figure 1. Mice can learn to discriminate LED pulse count using activity evoked in barrel cortex

(A) Opsin expression in barrel cortex. Left, widefield view of cranial window showing viral injection sites and LED; center, two-photon image of cranial window (green, GCaMP6s fluorescence; red, mScarlet fluorescence); right, higher magnification two-photon image. (B) Mice must lick the right (red) lickport following presentation of 7 or 9 LED pulses or the left (blue) lickport following presentation of 0,1 , or 3 pulses to obtain reward. 5 pulses are randomly rewarded. A spectrally matched masking flash LED was used throughout.

(C) Task timing during individual trial (Methods).

(D) Example photostimulation pulse permutations.

(E) Performance during learning. Top, task performance as a function of training day for one animal, with each dot indicating the rolling average of 61 trials centered on the shown trial (Black, days where peak d-prime $\geq 1.5$; grey, d-prime $<1.5$ ); bottom, pulse count progression.

(F) Task performance as a function of training day for individual animals. Colored, animals expressing opsin; grey, animals with no opsin.

(G) Right lick probability as a function of pulse count. Grey, individual animals; black, mean \pm standard error of the mean (SEM). 
bioRxiv preprint doi: https://doi.org/10.1101/2021.12.17.473191; this version posted December 20, 2021. The copyright holder for this preprint (which was not certified by peer review) is the author/funder, who has granted bioRxiv a license to display the preprint in perpetuity. It is made available under aCC-BY-NC-ND 4.0 International license.

(H) Lesion of opsin-expressing tissue. Left, coronal section following laser lesion (box, lesion extent; green, GCaMP6s fluorescence); right, task performance before and after lesion. Grey, average performance over 3 sessions pre- and post-lesion for individual animals; black: mean \pm SEM. 
Pancholi et al., Figure 2

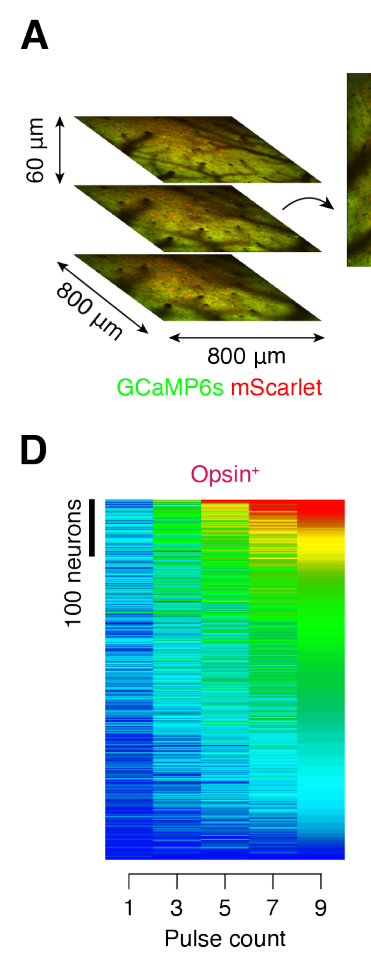

$\mathbf{F}$

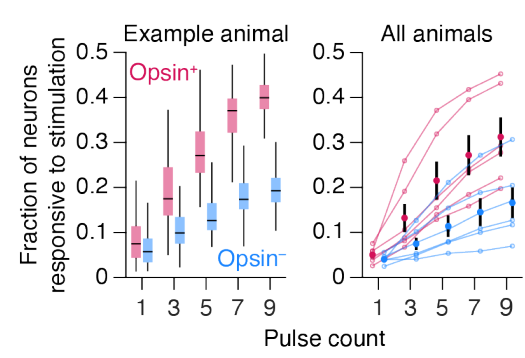

B
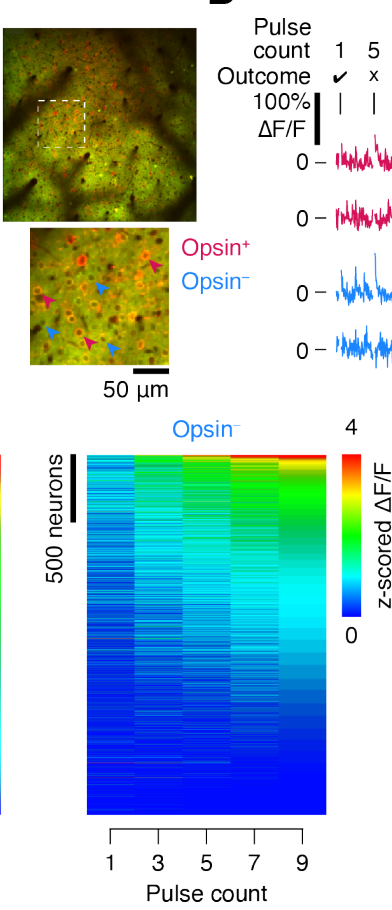

G

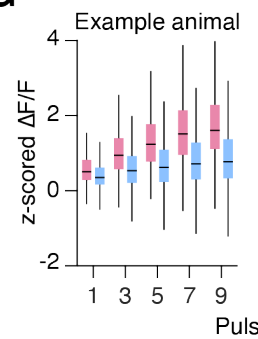

E

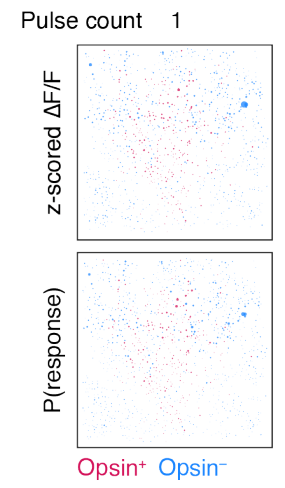

$\mathbf{H}$
C

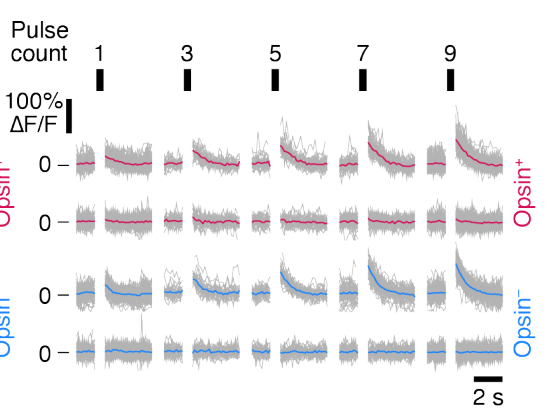
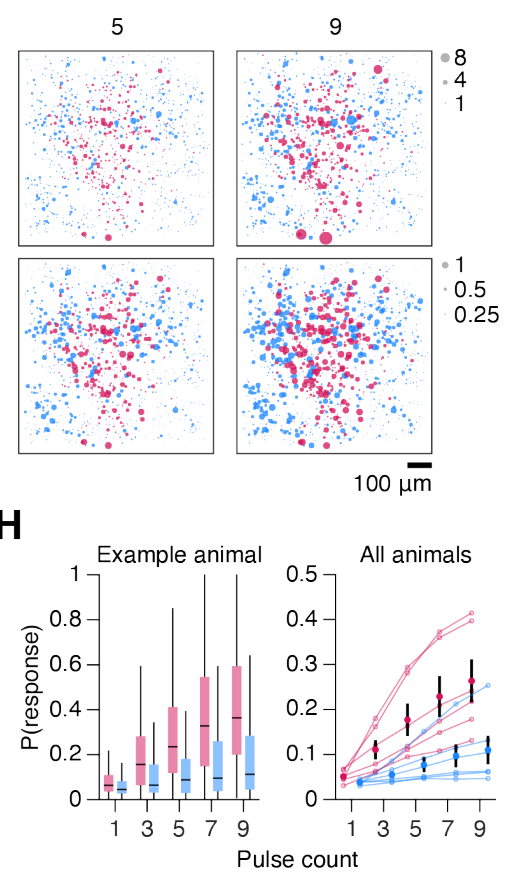

Figure 2. Volumetric two-photon imaging in behaving animals shows graded responses to photostimulation

(A) Volumetric two-photon imaging. Left, three imaging planes (800-by-800 $\mu \mathrm{m}, 60 \mu \mathrm{m}$ inter-plane distance) imaged simultaneously at $7 \mathrm{~Hz}$; right, example plane with example opsin-expressing and opsin non-expressing neurons (arrows).

(B) Photostimulation-evoked $\Delta \mathrm{F} / \mathrm{F}$ traces for 4 neurons across 10 consecutive trials. Vertical bars indicate stimulation epochs. Trial outcome indicates whether the animal made a correct (check) or incorrect (cross) behavioral response.

(C) $\Delta F / F$ traces for the same 4 neurons in (B) showing mean response to a particular pulse count over a single session. Grey, individual trials; color; mean.

(D) Response amplitudes (z-scored $\Delta \mathrm{F} / \mathrm{F}$ ) of all opsin-expressing and opsin nonexpressing neurons from one animal sorted by responses to 9 pulse stimulus.

(E) Spatial map of neural responses from one imaging plane. Size of dot indicates either response amplitude (top) or response probability (bottom) to 1, 5, or 9 stimulus pulses. 
bioRxiv preprint doi: https://doi.org/10.1101/2021.12.17.473191; this version posted December 20, 2021. The copyright holder for this preprint (which was not certified by peer review) is the author/funder, who has granted bioRxiv a license to display the preprint in perpetuity. It is made available under aCC-BY-NC-ND 4.0 International license.

(F) Fraction of neurons responsive to stimulation as a function of pulse count. Top, example animal; bottom, all animals (small dot, median; large dot, grand mean; error bars, SEM).

(G) Same as (F) for response amplitude as a function of pulse count (H) Same as (F) for response probability as a function of pulse count 
Pancholi et al., Figure 3

A

C

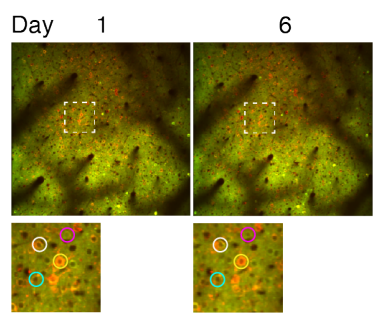

GCaMP6s mScarlet

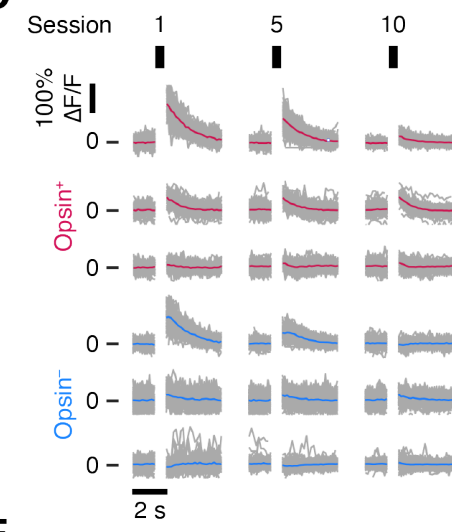

E

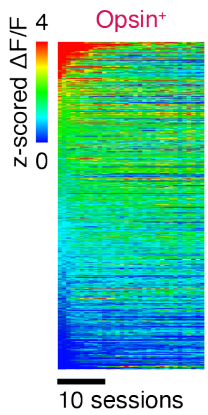

G

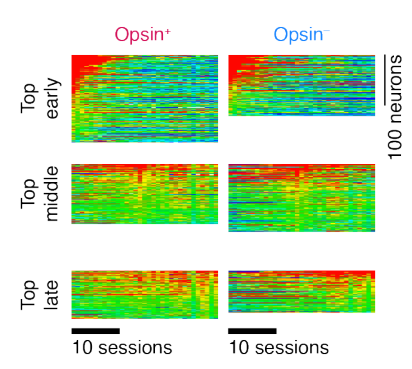

Opsin

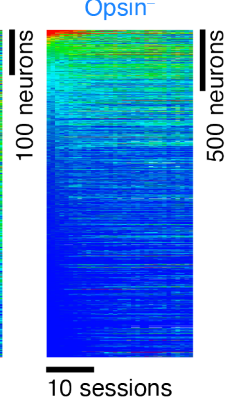

22
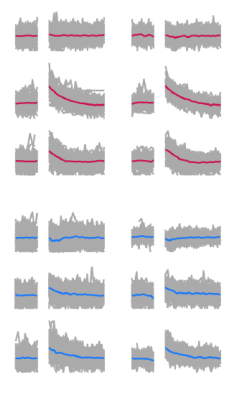

B

$\mathbf{F}$

D

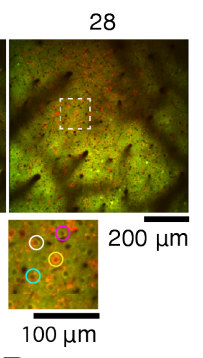

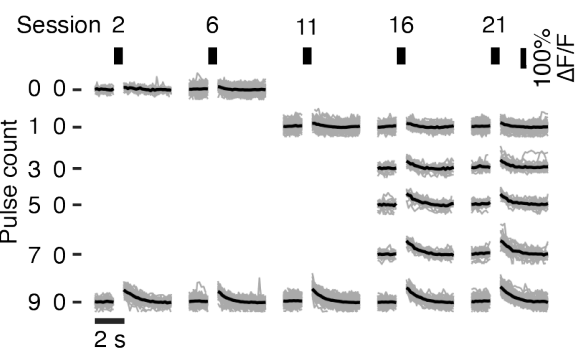
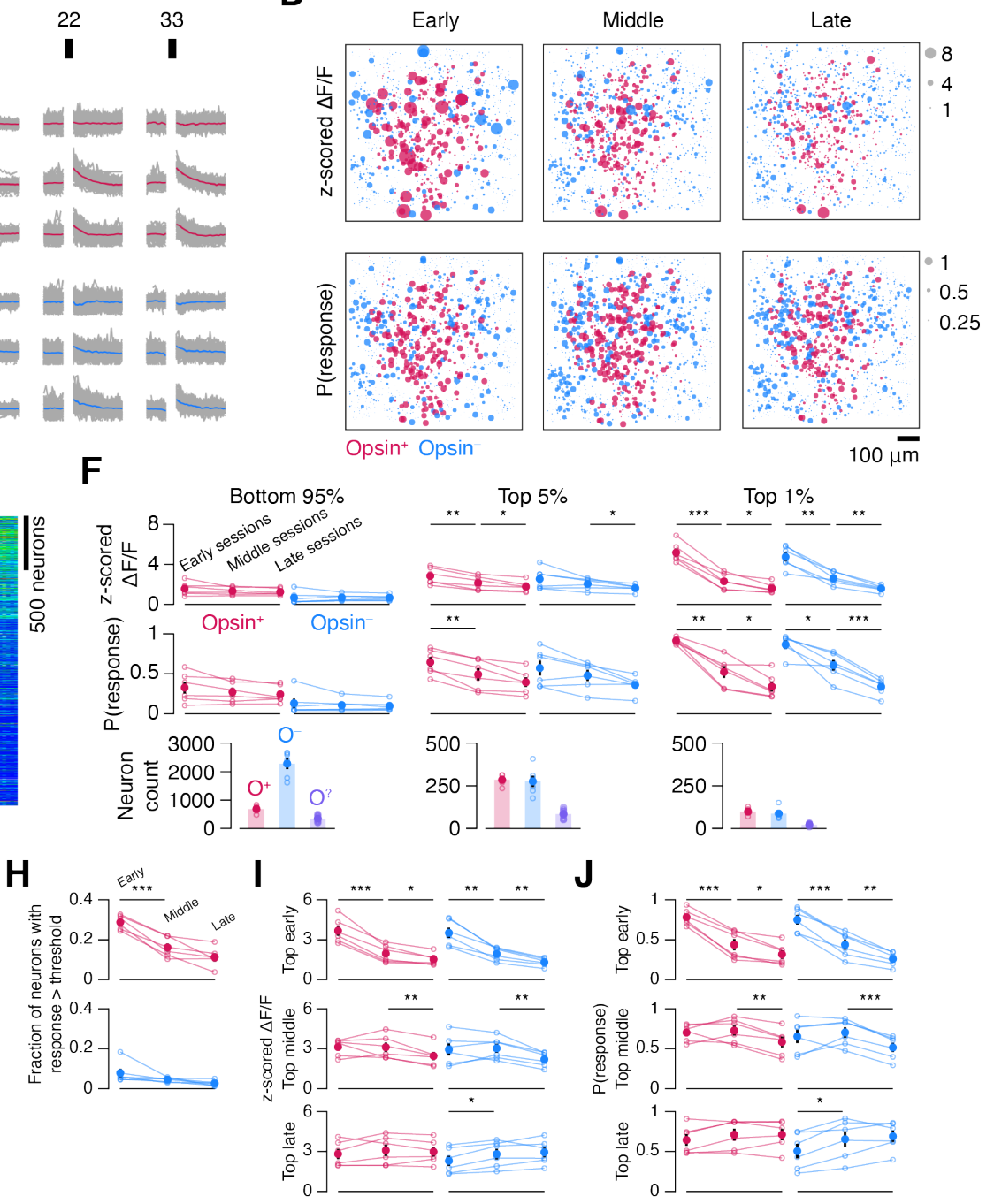

Figure 3. Chronic two-photon imaging reveals distinct neural dynamics across training

(A) Imaging plane across multiple days.

(B) Photostimulus responses of an example neuron across training to each pulse count. Vertical bars indicate stimulation epochs during which the PMT was shuttered. Grey, individual trials; black, mean. 
(C) Photostimulus responses of example neurons on 9 pulse trials across training. Top three rows: opsin-expressing neurons with decreasing (top), stable (middle), and increasing (bottom) responses across sessions. Bottom three rows: same for three opsin non-expressing neurons. Grey, individual trials; color, mean.

(D) Spatial maps of neurons from one imaging plane at three time points along the training progression. Size of dot indicates either response amplitude (top) or response probability (bottom).

(E) Response amplitude across sessions for all opsin-expressing and opsin nonexpressing neurons from one animal. Neurons are sorted by the mean response amplitude over the first three training sessions.

(F) Aggregate responsiveness across training. Left, bottom $95 \%$ of neurons by responsiveness (red, opsin-expressing; blue, opsin non-expressing). Each point indicates an animal's median response amplitude (top; z-scored $\Delta F / F$ ) or probability of response (middle) across three early, middle, or late sessions. Large dot, grand mean across animals. Error bars, SEM. Bottom, the total number of opsin-expressing $\left(\mathrm{O}^{+}\right)$, opsin nonexpressing $\left(\mathrm{O}^{-}\right)$, and opsin-ambiguous $\left(\mathrm{O}^{\text {? }}\right.$ ) neurons. Center, same as left panel but for neurons in the top 5\% of response amplitudes (Methods). Right, same as left panel but for neurons in the top percentile of response amplitudes .

(G) Response amplitude across sessions for neurons in the top $5 \%$ of response amplitudes in early, middle, or late sessions from one animal.

$(\mathrm{H})$ The fraction of highly responsive, opsin-expressing (top, red) neurons and opsin nonexpressing (bottom, blue) neurons. Small dot, animal median; large dot, grand mean. Error bars, SEM.

(I) Response amplitudes across training for highly responsive opsin-expressing and opsin non-expressing neurons selected in early, middle, or late sessions.

(J) Same as (I) for response probability.

${ }^{\star} p<0.05,{ }^{* *} p<0.01,{ }^{* *} p<0.001$, Bonferroni-adjusted paired t-tests; unlabeled, $p \geq 0.05$. 
Pancholi et al., Figure 4

A

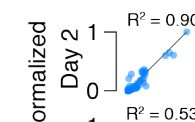

$$
\begin{aligned}
& \text { 는 } 1 \mathrm{R}^{2}=0.53
\end{aligned}
$$

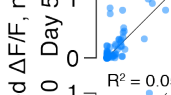

$$
\begin{aligned}
& \text { 인 }{ }^{1}{ }^{R^{2}=0.05} \\
& \text { N } \\
& \text { Day } 1 \\
& \text { Z-scored } \Delta F / F
\end{aligned}
$$

$\mathbf{F}$

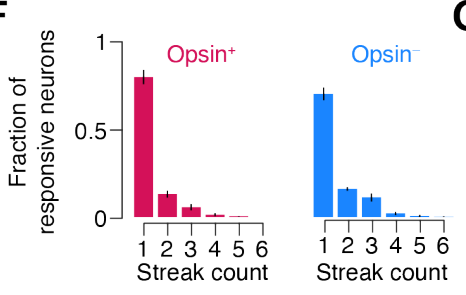

B

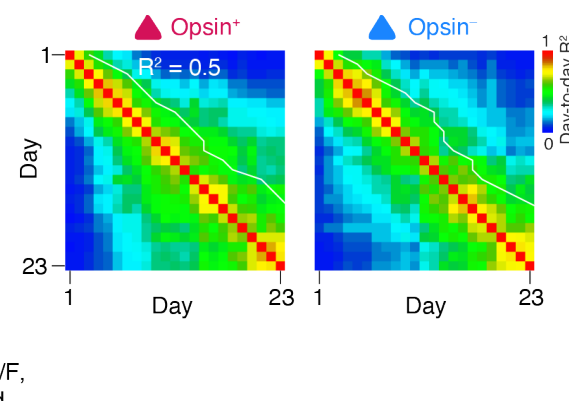

G

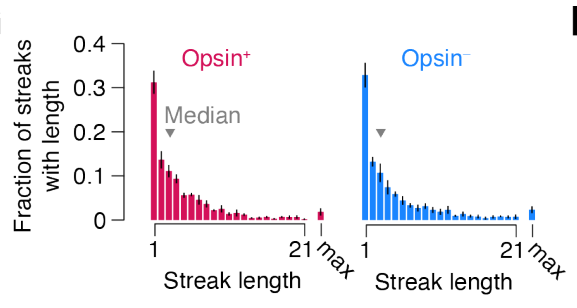

C D

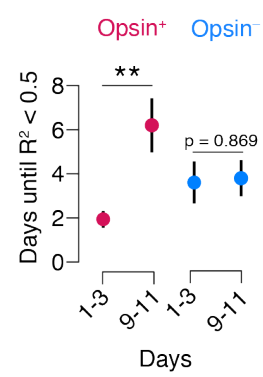

H

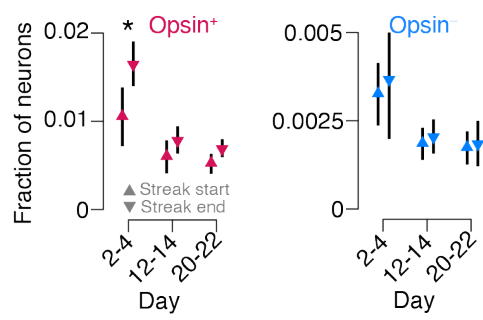

Figure 4. Turnover in neural responsiveness to photostimulation

(A) Correlation between response amplitude on various days with response amplitude on day 1 , with each dot representing a neuron that was responsive at some point during training.

(B) Mean correlation responsive neuron z-scored $\Delta F / F$ vectors between days across all mice for the first 23 days of imaging. White line indicates $R^{2}$ of 0.5 .

(C) Number of days from given starting point until $R^{2}$ between responsive neuron $z$-scored $\Delta \mathrm{F} / \mathrm{F}$ vectors for the compared days drops below 0.5 . Red, opsin-expressing neurons; blue, opsin non-expressing neurons. Point, mean across mice $(n=6)$. Error bars, SEM. ${ }^{* *} p<0.01$, paired t-test comparing day $1-3$ and day $9-11$.

(D) Probability of response for opsin-expressing (left) and opsin non-expressing (right) neurons that were highly responsive for at least one day (Methods). Neurons are sorted by responsiveness period start and end.

(E) Binary plot depicting responsiveness streaks for opsin non-expressing neurons from (D).

(F) Fraction of neurons that had a streak of a given length across mice (mean, $\mathrm{n}=6$; error bars, SEM). Left, opsin-expressing neurons; right, opsin non-expressing neurons.

(G) Distribution of streak length across all mice $(n=6)$ for opsin-expressing and opsin non-expressing neurons. Streaks of maximal length are grouped together for all animals. (H) Mean fraction of neurons starting (upward triangle) and ending (downward triangle) a streak on a given range of days. Left, opsin-expressing neurons; right, opsin nonexpressing neurons. ${ }^{*} p<0.05,{ }^{*} p<0.01$, Bonferroni-adjusted paired t-test comparing streak start and end fractions for that day range; unlabeled, $p \geq 0.05$. 
Pancholi et al., Figure 5

A

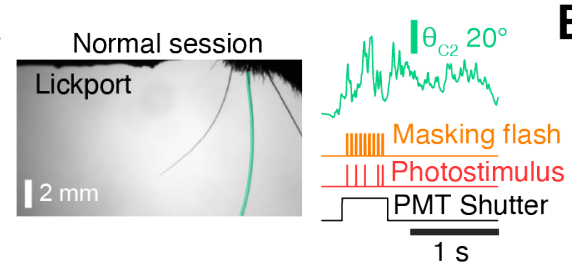

C

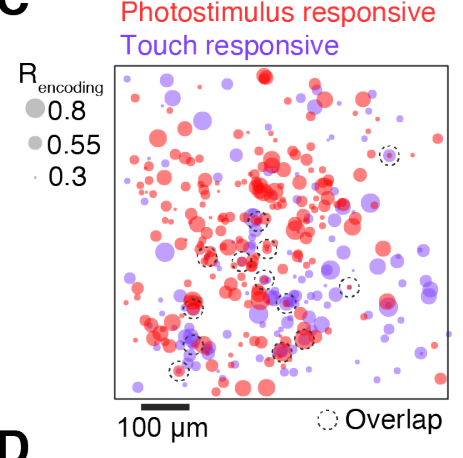

D

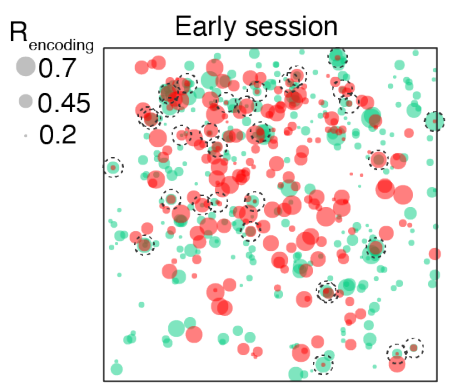

$E$

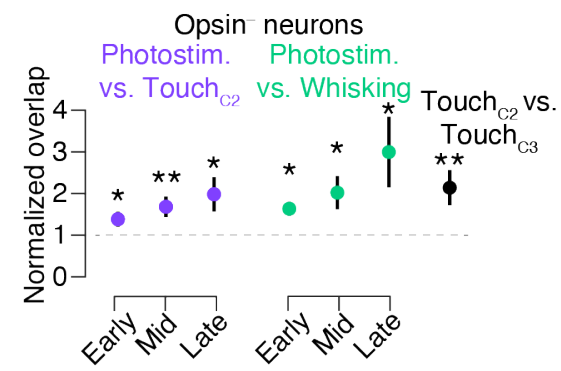

B

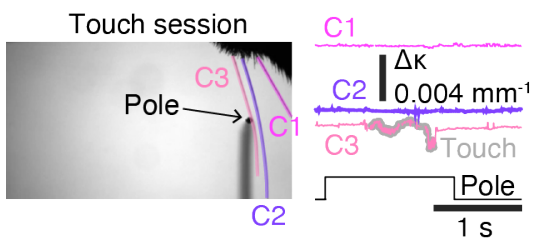

Photostimulus responsive

C2 touch responsive Whisking responsive

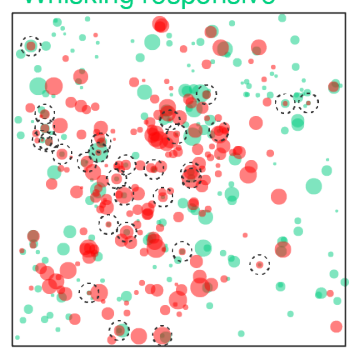

C3 touch responsive
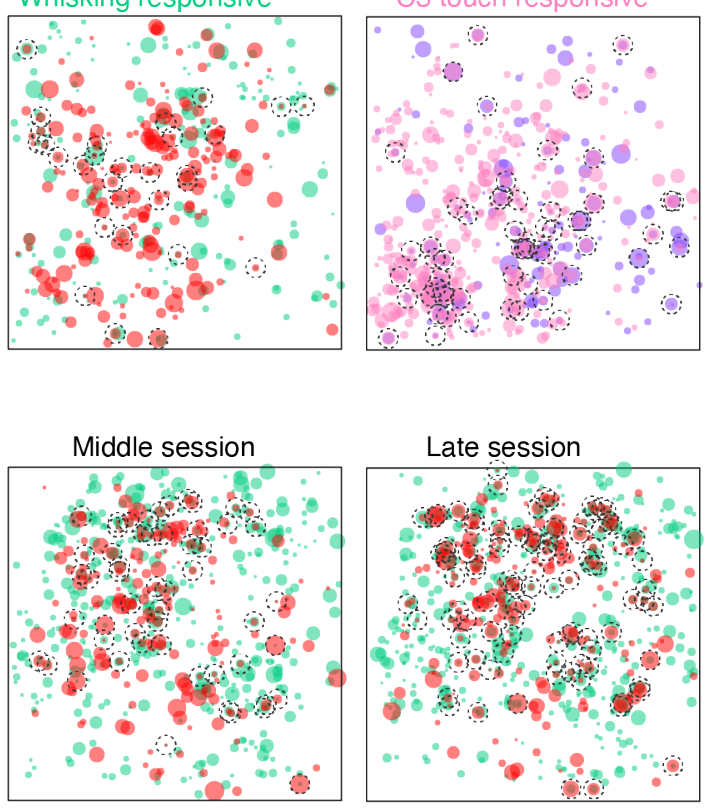

F

Opsin+ neurons

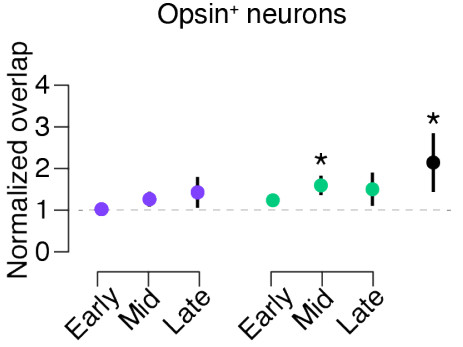

Figure 5. Interaction between photoresponsive and whisker-related populations

(A) Example whisker angle measurement during photostimulation task.

(B) Example whisker curvature measurement during task-free touch session (Methods).

(C) Maps comparing neural populations in an example plane. Left, photostimulus responsive (red) and touch responsive (C2; purple) populations; center, photostimulus responsive (red) and whisker movement responsive (green) populations; right, touch populations for the $\mathrm{C} 2$ and $\mathrm{C} 3$ whiskers. Dotted circles indicate neurons belonging to both representations. Circle size indicates encoding score (Methods). 
(D) Evolution of encoding score for photoresponsive (red) and whisker movement (green) representations. Dotted circles indicate neurons that belong to both representations.

(E) Overlap among populations over time for opsin non-expressing neurons, normalized to overlap when cell labels are randomized (Methods). Overlap was measured as the size of the intersection divided by the size of the union (Jaccard similarity). Left, photoresponsive vs. touch (C2 whisker) representations over time; right, photoresponsive vs. whisker movement representations. The overlap between $\mathrm{C} 2$ and $\mathrm{C} 3$ touch representations is shown for reference. Colored circle: mean across mice $(n=6)$. Error bars, SEM. ${ }^{*} p<0.05,{ }^{* *} p<0.01$, Bonferroni-adjusted t-test comparing observed values against a normal distribution with matched variance and mean of 1 ; unlabeled, $p \geq 0.05$. (F) As in (E) but for opsin-expressing neurons. 
Pancholi et al., Figure 6

A

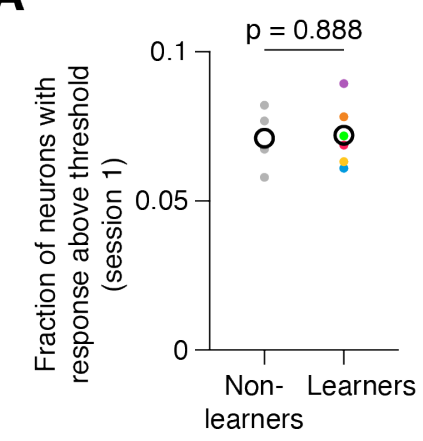

C

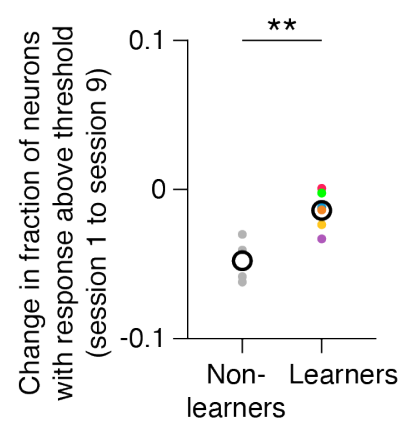

B

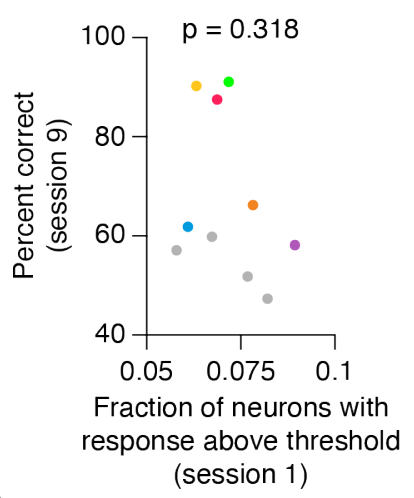

D

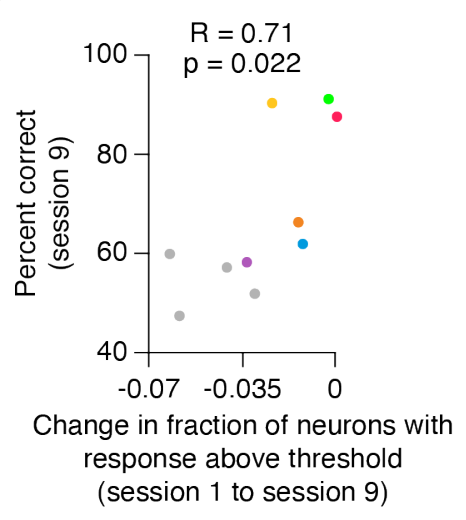

Figure 6. The influence of responsiveness dynamics on learning rate

(A) The fraction of highly responsive neurons (response amplitude in the top 5\% of response amplitudes; Methods) on day 1 for animals that did not learn the task (left, grey dots) and animals that did (right, colored dots). Open circles: mean.

(B) Relationship between performance on day 9 (final common day of training across all mice) and the fraction of highly responsive neurons on day 1 . P-value and $R$ given for Pearson correlation ( $\mathrm{n}=10$ mice).

(C) Change in the fraction of highly responsive neurons between days 1 and 9 for learners and non-learners. Symbols as in (A).

(D) Relationship between the change in fraction of highly responsive neurons from day 1 to 9 and animal performance on day 9. P-value and R given for Pearson correlation $(n=$ 10 mice). Symbols as in (B). 


\section{METHODS}

\section{RESOURCE AVAILABILITY}

Lead Contact. Further information and requests for resources and reagents should be directed to and will be fulfilled by the lead contact, Simon Peron (speron@nyu.edu).

Materials Availability. This study did not generate new unique reagents.

Data and Code Availability. Source code used in this paper will be made available at http://github.com/peronlab upon publication. Data from this paper will be made available in a public repository (DANDI archive) upon publication.

\section{EXPERIMENTAL MODEL AND SUBJECT DETAILS}

\section{Mouse lines}

Adult Ai162 (JAX 031562) X Slc17a7-Cre (JAX X 023527) (Daigle et al., 2018) mice (15 male, 1 female) were used throughout. These mice express GCaMP6s exclusively in cortical excitatory neurons in a tetracycline transactivator-dependent manner. To suppress expression during development, breeders were fed a diet that included doxycycline (625 mg/kg doxycycline; Teklad). Mice received doxycycline until weaning. All animal procedures were in compliance with protocols approved by New York University's University Animal Welfare Committee.

\section{METHOD DETAILS}

\section{Surgical preparation}

Mice (6-10 weeks old) were anesthetized with isoflurane during viral injections, surgical implantation, and LED placement (3\% induction, 1.5\% maintenance). A titanium headbar was attached to the skull with cyanoacrylate (Vetbond). A circular craniotomy $(3.5 \mathrm{~mm}$ diameter) was made in the left hemisphere over vS1 (center: $3.3 \mathrm{~mm}$ lateral, $1.7 \mathrm{~mm}$ posterior from bregma) using a dental drill (Midwest Tradition, FG 1/4 drill bit).

Following the craniotomy, a vector encoding the soma-localized opsin ChRmine and the red fluorophore mScarlet (AAV-8-CaMKIla-ChRmine-mScarlet-Kv2.1-WPRE, 2.48×1013 $\mathrm{vg} / \mathrm{mL}$, diluted 1:500 in 1X PBS; generously provided by Karl Deisseroth) was injected into vS1. A glass capillary (Wiretrol II, Drummond) was pulled using a micropipette puller (P-97, Sutter Instrument) and the tip beveled to $25^{\circ}$ with a tip diameter of $25 \mu \mathrm{m}$. The pipette was filled with mineral oil (M5904, Sigma-Aldrich) and $2 \mu \mathrm{L}$ of viral solution drawn into the tip using a plunger. Three, $100 \mathrm{~nL}$ viral injections were made $300 \mu \mathrm{m}$ below the dura and spaced $400 \mu \mathrm{m}$ apart in a triangle centered on the craniotomy. For each injection, the pipette was lowered into the brain at a rate of $300 \mu \mathrm{m} / \mathrm{min}$, there was a 1minute pause, virus was injected at a rate of $20 \mathrm{~nL} / \mathrm{min}$ using a hydraulic micromanipulator (Narishige MO-10), there was a 2 minute pause, and the pipette was withdrawn at a rate of $300 \mu \mathrm{m} / \mathrm{min}$ with an additional 1 minute pause at a depth of $150 \mu \mathrm{m}$ below the dura. Following injections, the dura was removed using a pair of fine forceps (Fine Science 
Tools) and a double-layer cranial window $(4.5 \mathrm{~mm}$ external diameter, $3.5 \mathrm{~mm}$ inner diameter; \#1.5 coverslip; adhered with Norland 61 UV glue) was placed over the craniotomy. The cranial window and headbar were affixed to the skull with dental acrylic (Orthojet, Lang Dental).

Following surgical recovery, mice were trimmed to whiskers $\mathrm{C} 1-3$ and placed on water restriction. To confirm that the area of opsin-expression fell within vS1, the location of the barrels corresponding to the $\mathrm{C} 1-3$ whiskers was identified by measuring touch-evoked GCaMP6s $\Delta \mathrm{F} / \mathrm{F}$ at coarse resolution (4X objective; $3 \times 3 \mathrm{~mm}$ field of view; Figures S1AC).

LED placement was performed 21-30 days after injection. LEDs $(590 \mathrm{~nm}, \mathrm{LXZ1-PL01}$, Lumileds) were fabricated by soldering $3 \mathrm{~cm}$ of polyurethane enameled copper wire (34 AWG) to each pad of the LED. A 2-pin, flat flex cable connector (Digikey) was then soldered to the free ends of the copper wire and secured with epoxy (Devcon). To waterproof the LED, a thin layer of clear nail polish (Sally Hansen) was applied to all surfaces of the LED. LEDs were then affixed to the animals' cranial windows. Following anesthesia, a small arc $\left(60^{\circ}\right)$ of dental cement was drilled away on the anterior-medial edge of the craniotomy to expose the edge of the cranial window. Cyanoacrylate was applied to the drilled area to ensure that the craniotomy remained sealed. A strip of electric tape was applied to the headbar to prevent conductance between the LED and the headbar. The LED was then placed at a $30^{\circ}$ angle on the edge of the cranial window with the most lateral edge of the LED $0.5-1 \mathrm{~mm}$ away from the center of the opsinexpressing area (Figures S1D and S1E). The medial edge of the LED and the copper wires were secured to the dental cement and the LED connector secured to the posterior edge of the headbar using cyanoacrylate. Waterproofing was confirmed by placing water on the cranial window and ensuring that no current passed between the water and the LED.

\section{Light propagation model}

The LED was modeled as an $8 \times 8$ grid of point sources positioned in water above the cranial window. A light ray from each point source would therefore pass through the following media: water, interface between water and window glass, glass, interface between glass and brain, and brain tissue. First, the fractional intensity of light emitted from the LED was computed using the LED radiation pattern. At each media interface, Fresnel's equations were used to calculate the fractional transmission of light through the interface. Within the brain, scattering of light was accounted for using empirical measurements (Li et al., 2019). The power density at different depths within the brain was computed by scaling the max power at the LED face (measured using a photodiode) by the fractional intensity, fractional transmission at each interface, and fraction of light remaining after scattering. This quantity was divided by the surface area of a half-sphere (inverse square law) to yield the predicted power density at a given location in the brain. Light radiation from each point source was treated independently and averaged to compute the final predicted power density.

\section{Photostimulation system}


Optogenetic stimulus delivery was controlled by a LabJack T7, with commands originating from a MATLAB user interface on a separate computer. The stimulation and masking flash LEDs were controlled using LED drivers (T-cube, ThorLabs) whose output currents were controlled by the LabJack. The signal to the stimulation LED was terminated using a 4-pin, flat flex cable connector (Digikey) that was mated to the LED connector on the animal during behavior. The masking flash consisted of 3 LEDs (595 nm, XPEBAM-L10000-00A01, Cree LED) that were spectrally matched to the stimulation LED and placed around the animal's face to illuminate the eyes.

\section{Behavior}

Mice were trained on an optogenetic pulse count discrimination task in which the number of light pulses from the stimulation LED was associated with a water reward from one of two lickports (Figure 1B). Each trial consisted of three epochs: stimulus (500 ms), delay (500 ms), and response (<2 s) (Figure 1C). During the stimulus epoch, between 0 and 9 light pulses $(5 \mathrm{~ms}, 20 \mathrm{~Hz})$ were presented from the stimulation LED. Pulses were randomly arranged in time among 9 time bins $(15 \mathrm{~ms}, 20 \mathrm{~Hz}$, stimulus pulse centered in each time bin) to prevent the animal from using temporal cues to solve the task (Figure 1D). Masking flash pulses $(15 \mathrm{~ms}, 20 \mathrm{~Hz})$ were presented throughout each time bin on all trials to prevent the animal from using visual cues to solve the task. The shutters to the two PMTs were closed $50 \mathrm{~ms}$ before the start of the first photostimulus time bin and opened $50 \mathrm{~ms}$ after the end of the last bin (total closure time: $505 \mathrm{~ms}$ ). Thus, the masking flash provided a visual cue for the onset of the stimulus epoch, whereas the shutter provided an auditory cue. Following the stimulus epoch, there was a $500 \mathrm{~ms}$ delay. An auditory response tone $(3 \mathrm{kHz}, 50 \mathrm{~ms})$ signaled the beginning of the response epoch. At this point, mice could indicate their decision by licking one of two lickports spaced $3 \mathrm{~mm}$ apart along the medial-lateral axis. The lickports were moved into reach only during the response epoch. Correct responses were rewarded with a 3-7 $\mu \mathrm{L}$ droplet of water which the animal could collect for 1-2 s. Incorrect responses were punished with immediate withdrawal of the lickport and a timeout. After the response epoch, there was a $7 \mathrm{~s}$ intertrial interval.

Training proceeded in a standard sequence. Following LED placement, water-restricted mice were handled and head-fixed to habituate animals to the behavioral apparatus. Animals were first trained on a photostimulus detection task in which 9 stimulation pulses predicted reward from the right lickport and 0 pulses predicted reward from the left lickport (Figures 1B and 1E). As mice surpassed our performance criterion (d-prime > 1.5) for more than 2 consecutive sessions for a given task stage, the task progressed as follows: 0 pulses (left) vs. 9 pulses (right); 1 pulse (left) vs. 9 pulses (right); 1 and 3 pulses (left) vs. 7 and 9 pulses (right); 1,3 , and 5 pulses (left) vs. 5,7 , and 9 pulses (right). 5 pulse trials were randomly rewarded between left and right. To maintain high performance on the final discrimination task and reduce biased licking, we modified the probability of presentation of each pulse count such that 1 and 9 pulse trials occurred 1-8x more frequently than intermediate pulse counts.

The behavioral task was controlled by a BPod state machine (Sanworks) and custom MATLAB software running on a behavioral computer (System 76 ). The auditory response 
tone was controlled by a low-latency, audio board (Bela). Lickport motion was controlled by a set of 3 motorized actuators (Zaber) and an Arduino. Licks were sensed using a custom electrical detection circuit (Janelia).

\section{Two-photon microscopy}

Calcium imaging was performed using a custom two-photon microscope (http://openwiki.janelia.org/wiki/display/shareddesigns/MIMMS). The microscope consisted of a $940 \mathrm{~nm}$ laser (Chameleon Ultra 2, Coherent), a Pockels cell (350-80-02, Conoptics), two galvanometer scanners (6SD11268, Cambridge Technology), a resonant scanner (6SC08KA040-02Y, Cambridge Technology), a 16x objective (N16XLWD-PF, Nikon), an emission filter for green fluorescence (FF01-510/84-30, Semrock), an emission filter for red fluorescence (FF01-650/60, Semrock), and two GaAsP PMTs (H10770PB40, Hamamatsu). Each PMT had an associated shutter (VS14S1T1, Vincent Associates) that was controlled by a voltage signal from the LabJack.

Imaging data was acquired using Scanimage (Vidrio Technologies). Three 800-by-800 $\mu \mathrm{m}$ imaging planes axially spaced $60 \mu \mathrm{m}$ apart were acquired at a rate of $\sim 7 \mathrm{~Hz}$. The objective was moved axially with a piezo (P-725KHDS, Physik Instrumente). Power was depth-adjusted by the acquisition software with an exponential length constant of 250 $\mu \mathrm{m}$.

Imaging data were processed on the NYU High Performance Computing cluster using a semi-automated software pipeline that included image registration, segmentation, neuropil subtraction, $\Delta F / F$ computation, and calcium event detection (Peron et al., 2015). After the first imaging day, motion-corrected mean images were collected for each plane and used as reference images on subsequent days. Alignment across days was performed as previously described (Huber et al., 2012).

Opsin expression was measured using mScarlet fluorescence collected in the red channel. For each pixel, we calculated a 'redness score', which consisted of the raw pixel value following linear de-mixing of the green channel from the red to remove cross-talk. For each neuron, an overall redness score was computed by taking the mean value for the de-mixed redness across its pixels. Finally, for each mouse, we manually selected a value above which neurons were considered opsin-expressing. Neurons with a redness below a second, lower threshold were considered opsin non-expressing. Neurons with an intermediate redness were considered ambiguous and were excluded from most analyses.

\section{Cortical lesions and histology}

Cortical lesions were performed with either an $800 \mathrm{~nm}$ (Chameleon Ultra 2, Coherent) or $1040 \mathrm{~nm}$ (Fidelity HP, Coherent) laser by focusing the laser at 200-300 $\mu \mathrm{m}$ depth for 12$40 \mathrm{~s}$ at $100 \%$ power ( $1.6 \mathrm{~W}$; Figure $1 \mathrm{H}$ ) at the infection site. Between 2 and 8 sites spaced 200-400 $\mu \mathrm{m}$ apart were targeted within the opsin-expressing area. Lesion success was visually confirmed by an increase in GCaMP6s fluorescence in the targeted area. In 2 animals, a second round of lesions was conducted the subsequent day because opsinexpression and evoked activity were still evident. Animals were awake but not performing the task during lesioning and were monitored for signs of distress or discomfort. Imaging 
data was not collected following lesioning. For histological analysis, animals were perfused after completion of training. Brains were sectioned using a microtome (Leica), mounted on glass slides, and imaged on a fluorescent light microscope (VS120, Olympus).

\section{Quantifying responsiveness}

Neurons were classified as responsive or non-responsive in every trial by comparing the post-stimulation $\Delta \mathrm{F} / \mathrm{F}$ to the baseline $\Delta \mathrm{F} / \mathrm{F}$. Baseline $\Delta \mathrm{F} / \mathrm{F}$ was selected to be the $\Delta \mathrm{F} / \mathrm{F}$ for the $\sim 5.5 \mathrm{~s}$ (39 frames) preceding shutter closure on each trial. The post-stimulation $\Delta \mathrm{F} / \mathrm{F}$ was calculated as the mean $\Delta \mathrm{F} / \mathrm{F}$ of the two frames immediately following shutter reopening. Neurons were considered photoresponsive on a given trial if the poststimulation $\Delta \mathrm{F} / \mathrm{F}$ exceeded the $97.5^{\text {th }}$ percentile or fell below the $2.5^{\text {th }}$ percentile of the distribution of baseline $\Delta F / F$ values across all trials in a session.

To compare responses between neurons and across sessions, we normalized the poststimulation $\Delta \mathrm{F} / \mathrm{F}$ using $\mathrm{z}$-scored normalization. For each session, we computed the mean $(\mu)$ and standard deviation $(\sigma)$ of the baseline $\Delta F / F$ over all trials for a neuron and then $z-$ scored each trial's post-stimulation $\Delta \mathrm{F} / \mathrm{F}$.

Highly responsive neurons were identified by first computing the mean z-scored $\Delta \mathrm{F} / \mathrm{F}$ over 9-pulse trials for each neuron across each session. A cross-neuron and crosssession distribution of mean z-scored $\Delta F / F$ values was constructed. Neurons were identified as highly responsive if they exceeded either the $95^{\text {th }}$ or $99^{\text {th }}$ percentiles of this distribution on at least one session of the sessions being considered.

\section{Turnover analysis}

We analyzed streaks (Fig. 4C-H) of responsiveness by first selecting neurons that were highly responsive on at least 1 day (response probability $>0.5$ ). We then applied a further threshold (response probability $>0.1$ ) to identify periods during which the neuron was active, filling gaps that were only a day long. For the analysis of streak start and end (Fig. $\mathbf{4 H}$ ), we only considered streaks starting on day 2 or later and ending on or prior to the penultimate day.

\section{Optical microstimulation encoding model}

Neurons were fit to an encoding model that predicted neural activity from a timeseries specifying the number of optogenetic pulse delivered on a given trial (Fig. S9A). The model attempts to predict calcium transients $(\Delta F / F)$ for each neuron. The model consists of an indicator kinetics kernel (defined by $\tau_{\text {rise }}$ and $\tau_{\text {decay }}$ ) along with an amplitude term that depends on the number of pulses delivered on a given trial. By convolving the indicator kinetics kernel with a timing vector in which each pulse count has a unique and specific amplitude, the model produces a $\Delta \mathrm{F} / \mathrm{F}$ vector with the same time base as the actual data. The kinetic terms, $\tau_{\text {rise }}$ and $\tau_{\text {decay }}$ were constrained based on the known physiological range (Chen et al., 2013): $\tau_{\text {rise }}, 100 \mathrm{~ms}$ to $500 \mathrm{~ms}$; $\tau_{\text {decay }}, 1 \mathrm{~s}$ to $5 \mathrm{~s}$.

The model was fit with 5-fold cross validation using block coordinate descent and a meansquare-error cost function minimizing the difference between model-predicted $\Delta \mathrm{F} / \mathrm{F}$ and actual $\Delta \mathrm{F} / \mathrm{F}$. During cross-validation, data was partitioned by randomly drawing 5 disjoint 
but interdigitated and equal sized sets of trials; individual trials were not broken up. We classified neurons by using the Pearson correlation of the model-predicted and actual $\Delta \mathrm{F} / \mathrm{F}$ traces for specific trial types. Neurons were considered photostimulation-responsive if this correlation exceeded 0.15 .

\section{Whisker videography and vibrissal representation analysis}

Mice were trimmed to a single row $(C)$ on the first day of training, with regular trimmings on subsequent days. Whisker video was acquired using custom MATLAB (MathWorks) software from a CMOS camera (Ace-Python 500, Basler) running at $400 \mathrm{~Hz}$ and $640 \mathrm{x}$ 352 pixels and using a telecentric lens (TitanTL, Edmund Optics). Illumination was provided by a pulsed $940 \mathrm{~nm}$ LED (SL162, Advanced Illumination). $7 \mathrm{~s}$ of each trial were recorded, starting $500 \mathrm{~ms}$ prior to shutter closure and including both the microstimulation and response periods. Data was processed on NYU's High Performance Computing (HPC) cluster. First, candidate whiskers were detected using the Janelia Whisker Tracker(Clack et al., 2012). Next, whisker identity was refined and assessed across a single session using custom MATLAB software (Peron et al., 2020; Peron et al., 2015). Following whisker assignment, the angle $(\theta)$ for one whisker (typically C2) was calculated, with protraction yielding more positive $\theta$ values, by convention.

For each mouse, a single, separate task-free session was employed to assess touch sensitivity, typically near the middle of training. During this session, a $0.5 \mathrm{~mm}$ diameter pole was brought into the whisker field and moved against one of the three largest whiskers (C1-3). For these sessions, change in curvature, $\Delta K$, was calculated relative a resting angle-dependent baseline curvature value obtained during periods when the pole was out of reach. This was followed by automatic touch detection. Touch assignment was manually curated using a custom MATLAB user interface (Peron et al., 2020).

Touch and whisking classification was performed using a per-neuron generalized linear model (GLM) that predicted $\Delta \mathrm{F} / \mathrm{F}$ from either $\theta$ (whisking neurons) or $\Delta K$ (touch neurons), described extensively elsewhere (Ahrens et al., 2008; Peron et al., 2020; Peron et al., 2015). The whisking model fit excluded the $\sim 2.5 \mathrm{~s}$ starting $1 \mathrm{~s}$ prior to shutter closure and ending $1 \mathrm{~s}$ following shutter closure, thereby excluding any photostimulation-related whisker movements. The touch fit included all timepoints. An encoding score was assigned to each neuron by computing the Pearson correlation between the modelpredicted $\Delta F / F$ and actual $\Delta F / F$. Neurons for which this value exceeded 0.15 were assigned to the touch and/or whisking representations. Whisking scores were computed for each day of imaging, whereas only a single touch score was computed per neuron.

\section{Overlap analysis}

Overlap was computed as the Jaccard similarity between two representations selected according to the aforementioned 0.15 encoding score criterion. This is defined as the size of the intersection of two sets divided by the size of their union. To compute the shuffled overlap for two representations containing $n_{A}$ and $n_{B}$ neurons, we drew $n_{A}$ and $n_{B}$ neurons with replacement from the population in question (opsin-expressing or opsin nonexpressing). The normalized overlap is the overlap measured for the actual representations divided by the median of 100 shuffled overlaps. 
bioRxiv preprint doi: https://doi.org/10.1101/2021.12.17.473191; this version posted December 20, 2021. The copyright holder for this preprint

(which was not certified by peer review) is the author/funder, who has granted bioRxiv a license to display the preprint in perpetuity. It is made available under aCC-BY-NC-ND 4.0 International license.

\section{Statistical analysis}

For comparisons between samples, two-tailed paired and unpaired t-tests were used. Where appropriate, the Bonferroni adjustment was applied to paired and unpaired t-tests to correct for multiple comparisons. For correlation tests, Pearson's correlation was used to identify a linear correlation coefficient $(\mathrm{R})$ and test for significance. 


\section{REFERENCES}

Afraz, S.R., Kiani, R., and Esteky, H. (2006). Microstimulation of inferotemporal cortex influences face categorization. Nature 442, 692-695.

Ahrens, M.B., Paninski, L., and Sahani, M. (2008). Inferring input nonlinearities in neural encoding models. Network 19, 35-67. 10.1080/09548980701813936.

Alejandre-García, T., Kim, S., Pérez-Ortega, J., and Yuste, R. (2020). Intrinsic excitability mechanisms of neuronal ensemble formation. bioRxiv, 2020.2007.2029.223966. 10.1101/2020.07.29.223966.

Audette, N.J., Bernhard, S.M., Ray, A., Stewart, L.T., and Barth, A.L. (2019). Rapid Plasticity of Higher-Order Thalamocortical Inputs during Sensory Learning. Neuron 103, 277-291 e274. 10.1016/j.neuron.2019.04.037.

Auffret, M., Ravano, V.L., Rossi, G.M.C., Hankov, N., Petersen, M.F.A., and Petersen, C.C.H. (2018). Optogenetic stimulation of cortex to map evoked whisker movements in awake head-restrained mice. Neuroscience 368, 199-213. 10.1016/j.neuroscience.2017.04.004.

Banerjee, A., Gonzalez-Rueda, A., Sampaio-Baptista, C., Paulsen, O., and RodriguezMoreno, A. (2014). Distinct mechanisms of spike timing-dependent LTD at vertical and horizontal inputs onto L2/3 pyramidal neurons in mouse barrel cortex. Physiological reports 2, e00271. 10.1002/phy2.271.

Barth, A.L., Gerkin, R.C., and Dean, K.L. (2004). Alteration of neuronal firing properties after in vivo experience in a FosGFP transgenic mouse. J Neurosci 24, 6466-6475.

Carrillo-Reid, L., Han, S., Yang, W., Akrouh, A., and Yuste, R. (2019). Controlling Visually Guided Behavior by Holographic Recalling of Cortical Ensembles. Cell 178, 447-457 e445. 10.1016/j.cell.2019.05.045.

Carrillo-Reid, L., Yang, W., Bando, Y., Peterka, D.S., and Yuste, R. (2016). Imprinting and recalling cortical ensembles. Science 353, 691-694. 10.1126/science.aaf7560.

Chen, J.L., Margolis, D.J., Stankov, A., Sumanovski, L.T., Schneider, B.L., and Helmchen, F. (2015). Pathway-specific reorganization of projection neurons in somatosensory cortex during learning. Nat Neurosci 18, 1101-1108. 10.1038/nn.4046.

Chen, T.W., Wardill, T.J., Sun, Y., Pulver, S.R., Renninger, S.L., Baohan, A., Schreiter, E.R., Kerr, R.A., Orger, M.B., Jayaraman, V., et al. (2013). Ultrasensitive fluorescent proteins for imaging neuronal activity. Nature 499, 295-300. 10.1038/nature12354. 
Chettih, S.N., and Harvey, C.D. (2019). Single-neuron perturbations reveal featurespecific competition in V1. Nature 567, 334-340. 10.1038/s41586-019-0997-6.

Chung, S., and Abbott, L.F. (2021). Neural population geometry: An approach for understanding biological and artificial neural networks. Curr Opin Neurobiol 70, 137-144. 10.1016/j.conb.2021.10.010.

Clack, N.G., O'Connor, D.H., Huber, D., Petreanu, L., Hires, A., Peron, S., Svoboda, K., and Myers, E.W. (2012). Automated tracking of whiskers in videos of head fixed rodents. PLoS Comput Biol 8, e1002591. 10.1371/journal.pcbi.1002591.

Clancy, K.B., Schnepel, P., Rao, A.T., and Feldman, D.E. (2015). Structure of a single whisker representation in layer 2 of mouse somatosensory cortex. J Neurosci 35, 39463958. 10.1523/JNEUROSCI.3887-14.2015.

Clopath, C., Bonhoeffer, T., Hubener, M., and Rose, T. (2017). Variance and invariance of neuronal long-term representations. Philos Trans R Soc Lond B Biol Sci 372. 10.1098/rstb.2016.0161.

Clopath, C., Busing, L., Vasilaki, E., and Gerstner, W. (2010). Connectivity reflects coding: a model of voltage-based STDP with homeostasis. Nat Neurosci 13, 344-352. $10.1038 / \mathrm{nn} .2479$.

Cohen, M.R., and Newsome, W.T. (2004). What electrical microstimulation has revealed about the neural basis of cognition. Curr Opin Neurobiol 14, 169-177.

Crochet, S., Poulet, J.F., Kremer, Y., and Petersen, C.C. (2011). Synaptic mechanisms underlying sparse coding of active touch. Neuron 69, 1160-1175. 10.1016/j.neuron.2011.02.022.

Daigle, T.L., Madisen, L., Hage, T.A., Valley, M.T., Knoblich, U., Larsen, R.S., Takeno, M.M., Huang, L., Gu, H., Larsen, R., et al. (2018). A Suite of Transgenic Driver and Reporter Mouse Lines with Enhanced Brain-Cell-Type Targeting and Functionality. Cell 174, 465-480 e422. 10.1016/j.cell.2018.06.035.

Dalgleish, H.W., Russell, L.E., Packer, A.M., Roth, A., Gauld, O.M., Greenstreet, F., Thompson, E.J., and Hausser, M. (2020). How many neurons are sufficient for perception of cortical activity? Elife 9. 10.7554/eLife.58889.

Deitch, D., Rubin, A., and Ziv, Y. (2021). Representational drift in the mouse visual cortex. Curr Biol 31, 4327-4339 e4326. 10.1016/j.cub.2021.07.062.

Desai, N.S., Rutherford, L.C., and Turrigiano, G.G. (1999). Plasticity in the intrinsic excitability of cortical pyramidal neurons. Nature Neuroscience 2, 515-520. 
Dhawale, A.K., Poddar, R., Wolff, S.B., Normand, V.A., Kopelowitz, E., and Olveczky, B.P. (2017). Automated long-term recording and analysis of neural activity in behaving animals. Elife 6. 10.7554/eLife.27702.

Feldman, D.E. (2009). Synaptic mechanisms for plasticity in neocortex. Annu Rev Neurosci 32, 33-55. 10.1146/annurev.neuro.051508.135516.

Finkelstein, A., Fontolan, L., Economo, M.N., Li, N., Romani, S., and Svoboda, K. (2021). Attractor dynamics gate cortical information flow during decision-making. Nat Neurosci 24, 843-850. 10.1038/s41593-021-00840-6.

Flesher, S.N., Downey, J.E., Weiss, J.M., Hughes, C.L., Herrera, A.J., Tyler-Kabara, E.C., Boninger, M.L., Collinger, J.L., and Gaunt, R.A. (2021). A brain-computer interface that evokes tactile sensations improves robotic arm control. Science 372, 831-836. 10.1126/science.abd0380.

Histed, M.H., Bonin, V., and Reid, R.C. (2009). Direct activation of sparse, distributed populations of cortical neurons by electrical microstimulation. Neuron 63, 508-522. S0896-6273(09)00545-5 [pii]

10.1016/j.neuron.2009.07.016.

Histed, M.H., and Maunsell, J.H. (2014). Cortical neural populations can guide behavior by integrating inputs linearly, independent of synchrony. Proc Natl Acad Sci U S A 111, E178-187. 10.1073/pnas.1318750111.

Histed, M.H., Ni, A.M., and Maunsell, J.H. (2012). Insights into cortical mechanisms of behavior from microstimulation experiments. Prog Neurobiol. 10.1016/j.pneurobio.2012.01.006.

Houweling, A.R., and Brecht, M. (2008). Behavioural report of single neuron stimulation in somatosensory cortex. Nature 451, 65-68. 10.1038/nature06447.

Huber, D., Gutnisky, D.A., Peron, S., O'Connor, D.H., Wiegert, J.S., Tian, L., Oertner, T.G., Looger, L.L., and Svoboda, K. (2012). Multiple dynamic representations in the motor cortex during sensorimotor learning. Nature 484, 473-478. 10.1038/nature11039.

Huber, D., Petreanu, L., Ghitani, N., Ranade, S., Hromadka, T., Mainen, Z., and Svoboda, K. (2008). Sparse optical microstimulation in barrel cortex drives learned behaviour in freely moving mice. Nature 451, 61-64. 10.1038/nature06445.

Kenet, T., Bibitchkov, D., Tsodyks, M., Grinvald, A., and Arieli, A. (2003). Spontaneously emerging cortical representations of visual attributes. Nature 425, 954-956. 10.1038/nature02078. 
Kim, T., Oh, W.C., Choi, J.H., and Kwon, H.B. (2016). Emergence of functional subnetworks in layer $2 / 3$ cortex induced by sequential spikes in vivo. Proc Natl Acad Sci U S A 113, E1372-1381. 10.1073/pnas.1513410113.

Knott, G.W., Quairiaux, C., Genoud, C., and Welker, E. (2002). Formation of dendritic spines with GABAergic synapses induced by whisker stimulation in adult mice. Neuron 34, 265-273.

Lefort, S., Tomm, C., Floyd Sarria, J.C., and Petersen, C.C. (2009). The excitatory neuronal network of the $\mathrm{C} 2$ barrel column in mouse primary somatosensory cortex. Neuron 61, 301-316. 10.1016/j.neuron.2008.12.020.

Li, N., Chen, S., Guo, Z.V., Chen, H., Huo, Y., Inagaki, H.K., Chen, G., Davis, C., Hansel, D., Guo, C., and Svoboda, K. (2019). Spatiotemporal constraints on optogenetic inactivation in cortical circuits. Elife 8. 10.7554/eLife.48622.

Litwin-Kumar, A., and Doiron, B. (2014). Formation and maintenance of neuronal assemblies through synaptic plasticity. Nat Commun 5, 5319. 10.1038/ncomms6319.

Luczak, A., Bartho, P., and Harris, K.D. (2009). Spontaneous events outline the realm of possible sensory responses in neocortical populations. Neuron 62, 413-425.

Marks, T.D., and Goard, M.J. (2021). Stimulus-dependent representational drift in primary visual cortex. Nat Commun 12, 5169. 10.1038/s41467-021-25436-3.

Marshel, J.H., Kim, Y.S., Machado, T.A., Quirin, S., Benson, B., Kadmon, J., Raja, C., Chibukhchyan, A., Ramakrishnan, C., Inoue, M., et al. (2019). Cortical layer-specific critical dynamics triggering perception. Science 365. 10.1126/science.aaw5202.

Matyas, F., Sreenivasan, V., Marbach, F., Wacongne, C., Barsy, B., Mateo, C., Aronoff, R., and Petersen, C.C. (2010). Motor control by sensory cortex. Science 330, 1240-1243. $330 / 6008 / 1240$ [pii]

10.1126/science.1195797.

O'Connor, D.H., Hires, S.A., Guo, Z.V., Li, N., Yu, J., Sun, Q.Q., Huber, D., and Svoboda, K. (2013). Neural coding during active somatosensation revealed using illusory touch. Nat Neurosci 16, 958-965. 10.1038/nn.3419.

Pandarinath, C., and Bensmaia, S.J. (2021). The science and engineering behind sensitized brain-controlled bionic hands. Physiol Rev. 10.1152/physrev.00034.2020.

Penfield, W., and Boldery, P. (1937). Somatic motor and sensory representation in the cerebral cortex of man as studied by electrical stimulation. Brain 60, 389-443.

Perez-Ortega, J., Alejandre-Garcia, T., and Yuste, R. (2021). Long-term stability of cortical ensembles. Elife 10. 10.7554/eLife.64449. 
Peron, S., Pancholi, R., Voelcker, B., Wittenbach, J.D., Olafsdottir, H.F., Freeman, J., and Svoboda, K. (2020). Recurrent interactions in local cortical circuits. Nature 579, 256-259. 10.1038/s41586-020-2062-x.

Peron, S.P., Freeman, J., lyer, V., Guo, C., and Svoboda, K. (2015). A Cellular Resolution Map of Barrel Cortex Activity during Tactile Behavior. Neuron 86, 783-799. 10.1016/j.neuron.2015.03.027.

Polikov, V.S., Tresco, P.A., and Reichert, W.M. (2005). Response of brain tissue to chronically implanted neural electrodes. J Neurosci Methods 148, 1-18. 10.1016/j.jneumeth.2005.08.015.

Polley, D.B., Kvasnak, E., and Frostig, R.D. (2004). Naturalistic experience transforms sensory maps in the adult cortex of caged animals. Nature 429, 67-71.

Poort, J., Khan, A.G., Pachitariu, M., Nemri, A., Orsolic, I., Krupic, J., Bauza, M., Sahani, M., Keller, G.B., Mrsic-Flogel, T.D., and Hofer, S.B. (2015). Learning Enhances Sensory and Multiple Non-sensory Representations in Primary Visual Cortex. Neuron 86, 14781490. 10.1016/j.neuron.2015.05.037.

Romo, R., Hernandez, A., Zainos, A., and Salinas, E. (1998). Somatosensory discrimination based on cortical microstimulation. Nature 392, 387-390. 10.1038/32891.

Rule, M.E., O'Leary, T., and Harvey, C.D. (2019). Causes and consequences of representational drift. Curr Opin Neurobiol 58, 141-147. 10.1016/j.conb.2019.08.005.

Rutkowski, R.G., and Weinberger, N.M. (2005). Encoding of learned importance of sound by magnitude of representational area in primary auditory cortex. Proc Natl Acad Sci U S A 102, 13664-13669. 10.1073/pnas.0506838102.

Sadeh, S., and Clopath, C. (2021). Excitatory-inhibitory balance modulates the formation and dynamics of neuronal assemblies in cortical networks. bioRxiv, 2021.2004.2015.439946. 10.1101/2021.04.15.439946.

Salzman, C.D., Britten, K.H., and Newsome, W.T. (1990). Cortical microstimulation influences perceptual judgements of motion direction. Nature 346, 174-177. 10.1038/346174a0.

Schoonover, C.E., Ohashi, S.N., Axel, R., and Fink, A.J.P. (2021). Representational drift in primary olfactory cortex. Nature 594, 541-546. 10.1038/s41586-021-03628-7.

Tanke, N., Borst, J.G.G., and Houweling, A.R. (2018). Single-Cell Stimulation in Barrel Cortex Influences Psychophysical Detection Performance. J Neurosci 38, 2057-2068. 10.1523/JNEUROSCI.2155-17.2018. 
Tyler, D.J. (2015). Neural interfaces for somatosensory feedback: bringing life to a prosthesis. Curr Opin Neurol 28, 574-581. 10.1097/WCO.0000000000000266.

Vogels, T.P., Sprekeler, H., Zenke, F., Clopath, C., and Gerstner, W. (2011). Inhibitory plasticity balances excitation and inhibition in sensory pathways and memory networks. Science 334, 1569-1573. 10.1126/science.1211095.

Weinberger, N.M. (2004). Specific long-term memory traces in primary auditory cortex. Nat Rev Neurosci 5, 279-290. 10.1038/nrn1366.

Weiss, J.M., Flesher, S.N., Franklin, R., Collinger, J.L., and Gaunt, R.A. (2019). Artifactfree recordings in human bidirectional brain-computer interfaces. J Neural Eng 16, 016002. 10.1088/1741-2552/aae748.

Wimmer, V.C., Broser, P.J., Kuner, T., and Bruno, R.M. (2010). Experience-induced plasticity of thalamocortical axons in both juveniles and adults. J Comp Neurol 518, 46294648. $10.1002 /$ cne.22483.

Yang, Y., and Zador, A.M. (2012). Differences in sensitivity to neural timing among cortical areas. J Neurosci 32, 15142-15147. 10.1523/JNEUROSCI.1411-12.2012.

Zhang, D., Yan, X., She, L., Wen, Y., and Poo, M.M. (2020). Global enhancement of cortical excitability following coactivation of large neuronal populations. Proc Natl Acad Sci U S A 117, 20254-20264. 10.1073/pnas.1914869117. 


\section{SUPPLEMENTARY MATERIALS}

\begin{tabular}{|l|l|l|l|l|l|l|}
\hline $\begin{array}{l}\text { Animal } \\
\text { ID }\end{array}$ & Sex & $\begin{array}{l}\text { Age at training } \\
\text { start (days) }\end{array}$ & $\begin{array}{l}\text { Opsin+ }^{+} \\
\text {cell count }\end{array}$ & $\begin{array}{l}\text { Opsin- } \\
\text { cell count }\end{array}$ & $\begin{array}{l}\text { Learning } \\
\text { status }\end{array}$ & $\begin{array}{l}\text { Opsin } \\
\text { expression }\end{array}$ \\
\hline $\mathbf{2 9 3 0 1 0}$ & M & 75 & 775 & 2593 & Learner & Yes \\
\hline $\mathbf{2 9 3 1 5 7}$ & M & 96 & 718 & 2679 & Learner & Yes \\
\hline $\mathbf{2 9 3 1 6 7}$ & M & 66 & 486 & 2413 & Learner & Yes \\
\hline $\mathbf{2 9 5 5 7 8}$ & M & 77 & 624 & 2640 & Learner & Yes \\
\hline $\mathbf{2 9 5 6 7 8}$ & M & 83 & 836 & 1621 & Learner & Yes \\
\hline $\mathbf{0 1 0 8 5 2}$ & M & 87 & 717 & 1778 & Learner & Yes \\
\hline & & & & & & \\
\hline $\mathbf{2 9 3 1 5 6}$ & M & 107 & 725 & 2530 & Non-learner & Yes \\
\hline $\mathbf{2 9 4 7 5 5}$ & M & 90 & 766 & 2360 & Non-learner & Yes \\
\hline $\mathbf{2 9 5 1 3 7}$ & M & 74 & 863 & 2183 & Non-learner & Yes \\
\hline $\mathbf{2 9 5 3 3 1}$ & M & 83 & 693 & 2178 & Non-learner & Yes \\
\hline & & & & & & \\
\hline $\mathbf{2 9 7 1 0 8}$ & M & 67 & N/A & N/A & Non-learner & No \\
\hline $\mathbf{2 9 7 1 0 9}$ & M & 67 & N/A & N/A & Non-learner & No \\
\hline $\mathbf{2 9 7 6 7 0}$ & M & 58 & N/A & N/A & Non-learner & No \\
\hline $\mathbf{2 9 7 0 2 3}$ & M & 81 & N/A & N/A & Non-learner & No \\
\hline $\mathbf{2 9 7 0 2 7}$ & F & 82 & N/A & N/A & Non-learner & No \\
\hline & & & & & & N/A \\
\hline $\mathbf{0 0 7 4 1 7}$ & M & N/A & 829 & 2731 & Yes \\
\hline
\end{tabular}

Table S1. List of animals, Related to Figure 1. All mice were transgenic Ai162 X Slc17a7-Cre (Daigle et al., 2018), expressing GCaMP6s in excitatory neurons. Non opsinexpressing controls were only used in Figure 1. Opsin-expressing non-learners were only used in Figure 6. One animal was never trained and used for calibration of photostimulation parameters in Figure S3. 
Pancholi et al., Figure S1

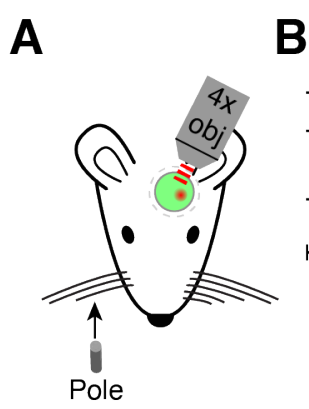

D

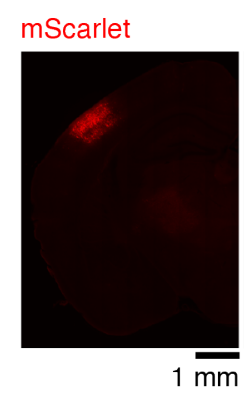

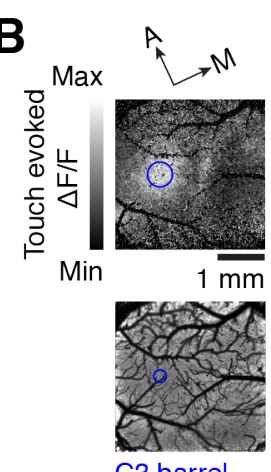

C3 barrel
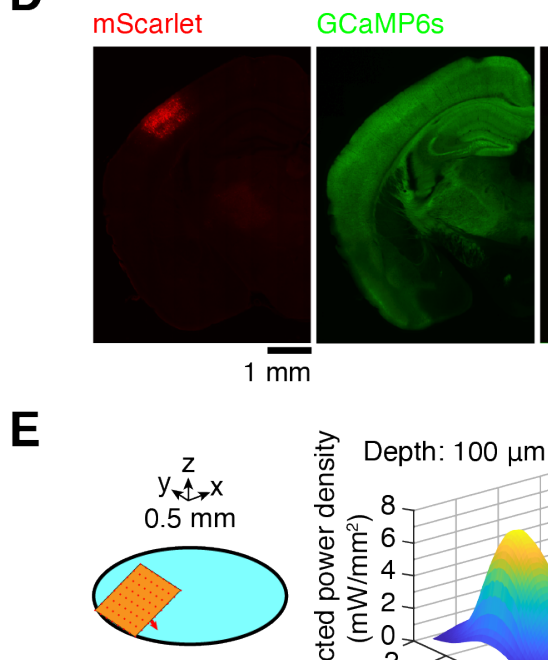

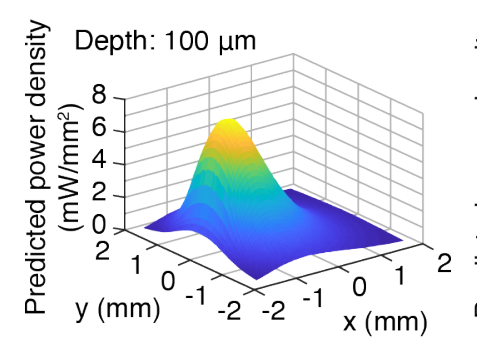

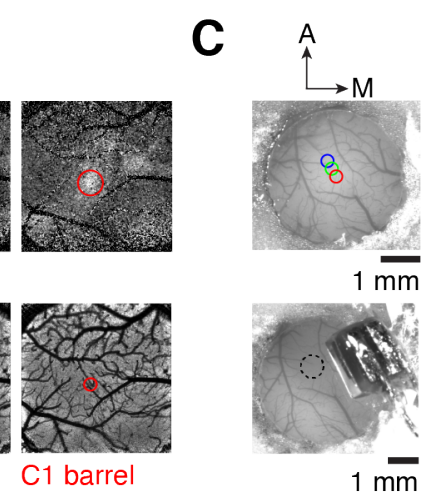

: opsin expression
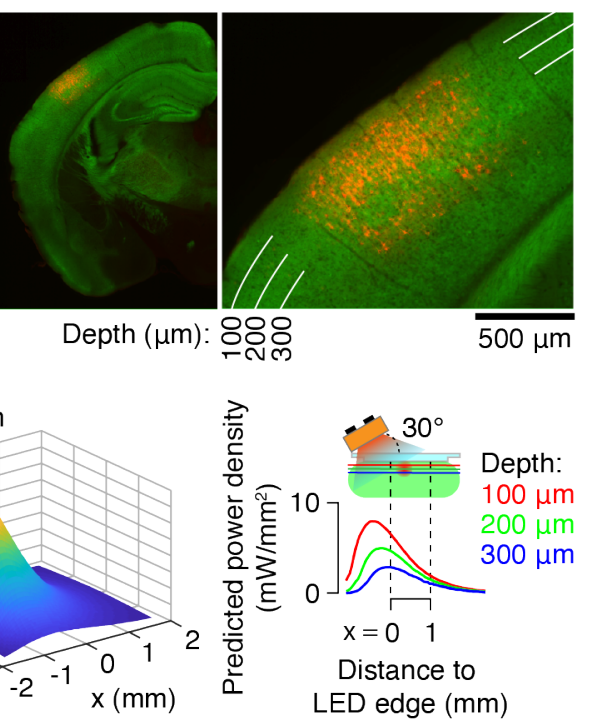

Figure S1, Related to Figure 1. Targeting single barrel for superficial opsin expression.

(A) Barrel mapping was conducted by driving a pole into whiskers $\mathrm{C} 1-3$ one at a time during widefield two-photon calcium imaging.

(B) Touch-evoked $\Delta F / F$ following whisker stimulation. Top, $\Delta F / F$ following touch by indicated whisker; bottom, structural image showing barrel locations.

(C) Cranial window prior to LED placement (top) and following LED placement (bottom). Colored circles indicate the identified barrel locations from (B), and the dotted circle indicates the site of opsin expression.

(D) Opsin expression. Left to right: expression of mScarlet, the fluorophore co-expressed with ChRmine; expression of GCaMP6s; overlay of GCaMP6s and mScarlet; magnified overlay.

(E) Simulation of light propagation in the opsin-expressing tissue (Methods). Left, geometric model of the LED and cranial window; center, predicted power density surface at a depth of $100 \mu \mathrm{m}$ in the brain; right, predicted power density at various depths in the brain and distances from the LED edge. 
Pancholi et al., Figure S2

A

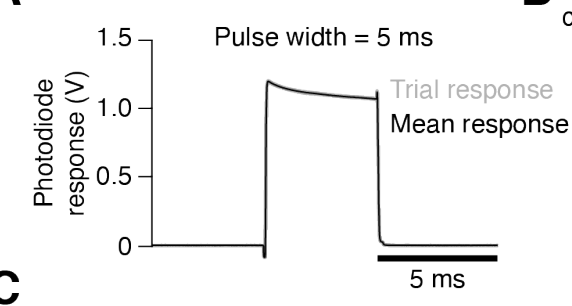

B LED IR

connector Objective filter Photodiode Objective filter Photodiode

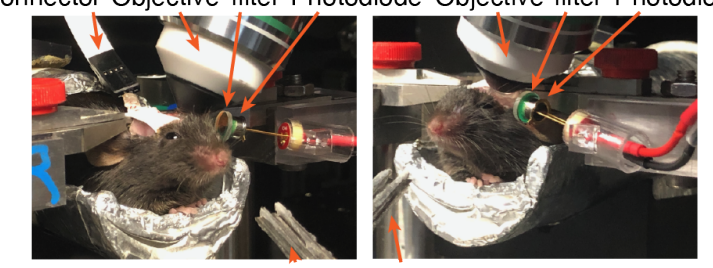

Pulse count 9999009900990099900090099

Lickport Lickport
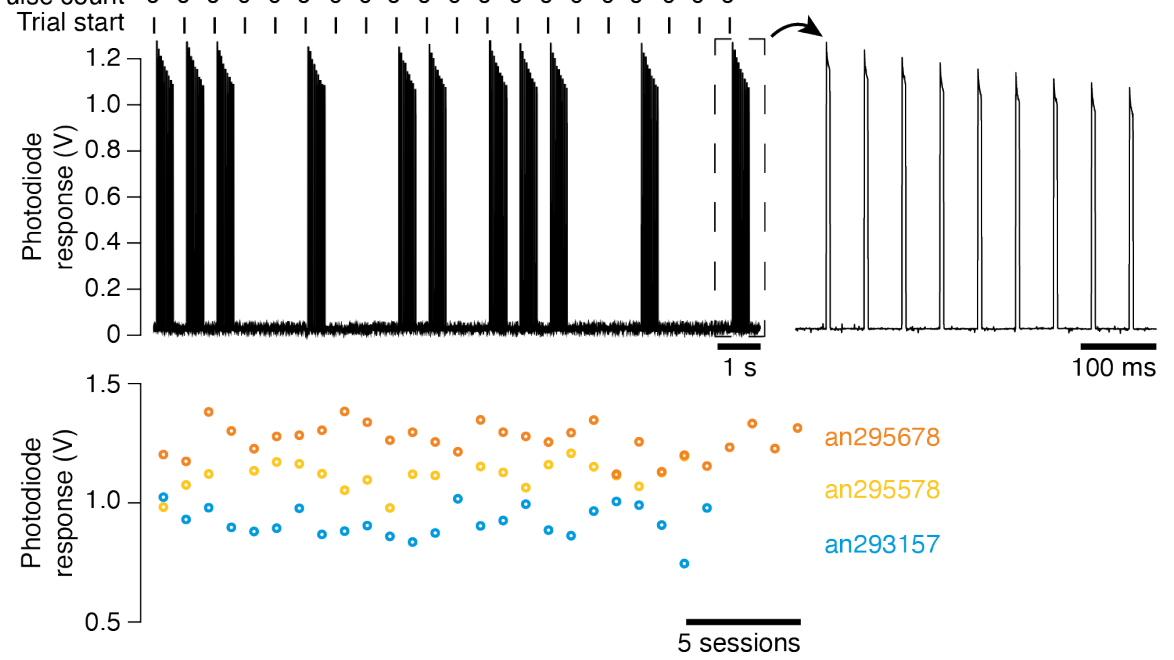

$\mathbf{E}$

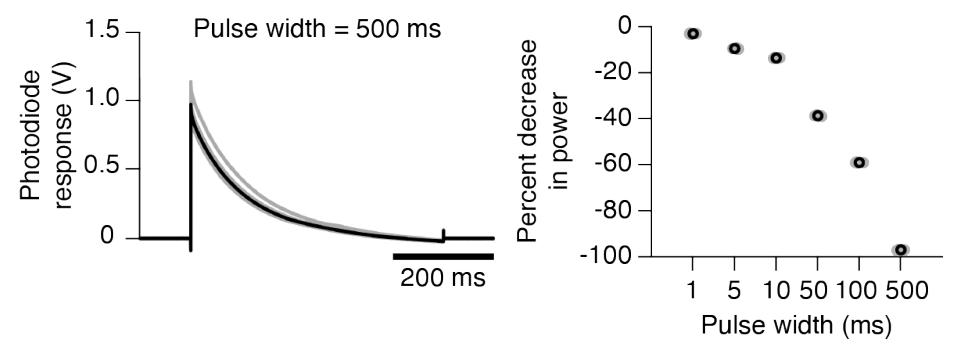

Figure S2, Related to Figure 1. LED is a robust and reproducible method of stimulation

(A) LED responses as measured by a standalone photodiode. 10 repetitions of a single 5 ms pulse at $100 \%$ power. Grey, individual repetitions; black, mean.

(B) Placement of photodiode used to measure LED output.

(C) Photodiode responses to LED stimulation across 20 consecutive trials in one behavioral session.

(D) Photodiode responses to 9 pulse trials across sessions for 3 animals. LED was tested for 10-20 trials at the conclusion of each behavioral session following removal of water for imaging objective.

(E) Photodiode responses to long duration pulses. Left, same as (A) for a $500 \mathrm{~ms}$ pulse; right, percent decrease in power between pulse start and pulse end for various pulse widths. Grey, individual repetitions; black, mean across repetitions. 
bioRxiv preprint doi: https://doi.org/10.1101/2021.12.17.473191; this version posted December 20, 2021. The copyright holder for this preprint (which was not certified by peer review) is the author/funder, who has granted bioRxiv a license to display the preprint in perpetuity. It is made available under aCC-BY-NC-ND 4.0 International license.

\section{Pancholi et al., Figure S3}

A

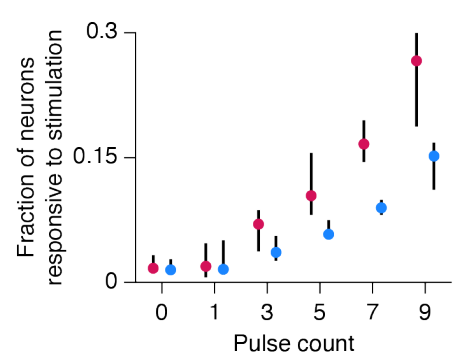

Stimulation parameters

Stimulation parameters
count $=$ variable, duration $=5 \mathrm{~ms}$, amplitude $=100 \%$, freq $=20 \mathrm{~Hz}$

B
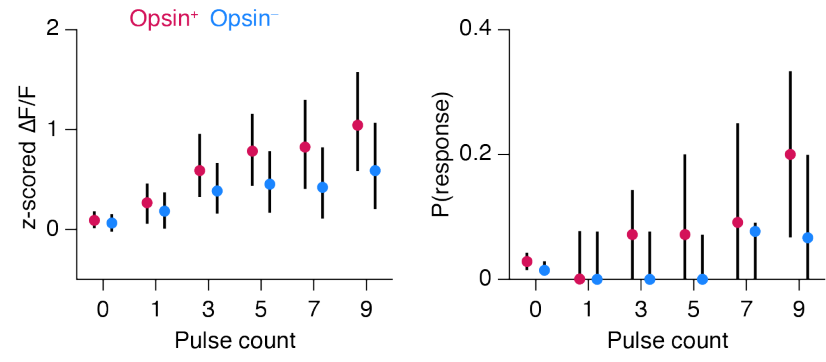

Stimulation parameters count $=5$ pulses, duration $=5 \mathrm{~ms}$, amplitude $=100 \%$, freq $=$ variable

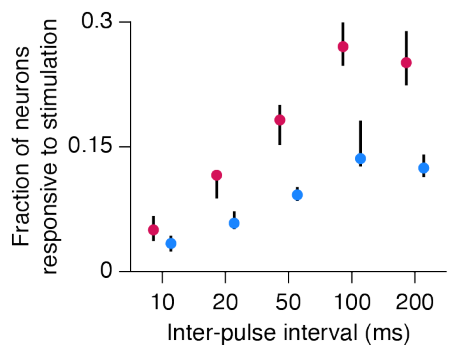

C

Stimulation parameters

count $=$ variable, duration $=$ variable, amplitude $=100 \%$, freq $=20 \mathrm{~Hz}$ Fraction of neurons
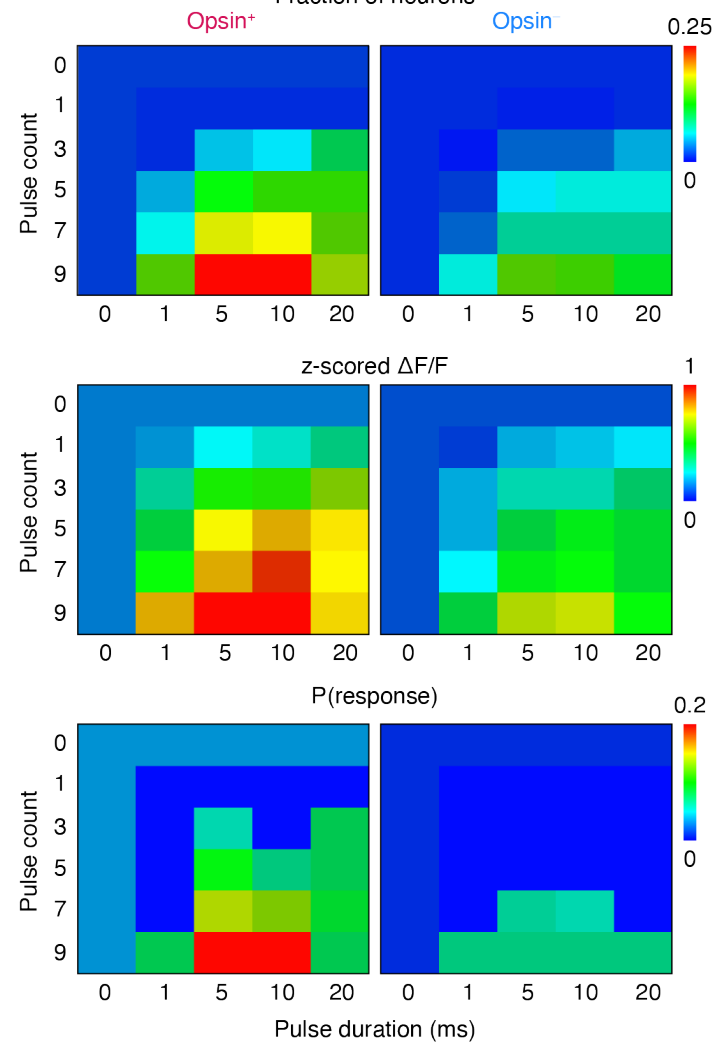
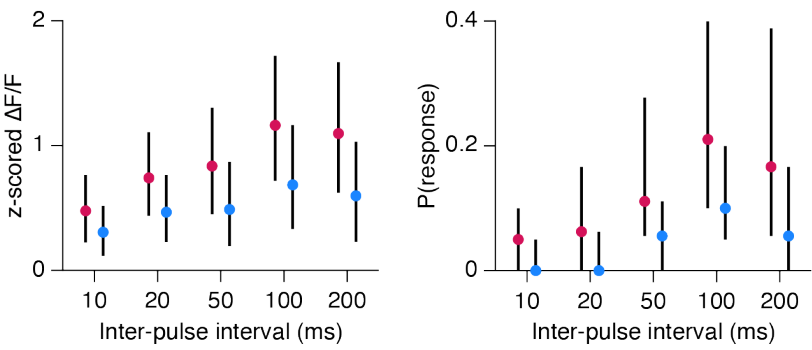

D

Stimulation parameters

count $=$ variable, duration $=5 \mathrm{~ms}$, amplitude $=$ variable, freq $=20 \mathrm{~Hz}$ Fraction of neurons
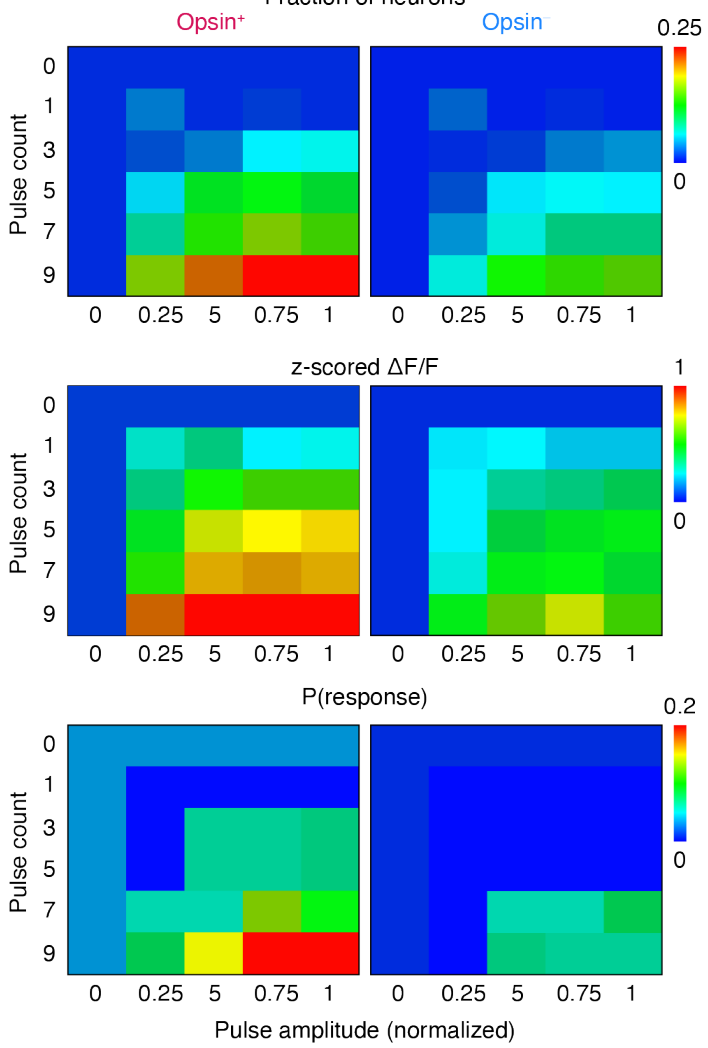
Figure S3, Related to Figure 1. Neural responsiveness varies as a function of stimulation parameters

(A) Fraction of neurons responding (left), response amplitude (center), and response probability (right) as a function of pulse count for opsin-expressing (red) and opsin nonexpressing (blue) neurons. Data are shown as median \pm inter-quartile range.

(B) Same as (A) except as a function of inter-pulse interval.

(C) Fraction of neurons responding (top), response amplitude (middle), and response probability (bottom) as function of both pulse count and pulse duration.

(D) Same as (C) except for pulse count and pulse amplitude. 
Pancholi et al., Figure S4

A

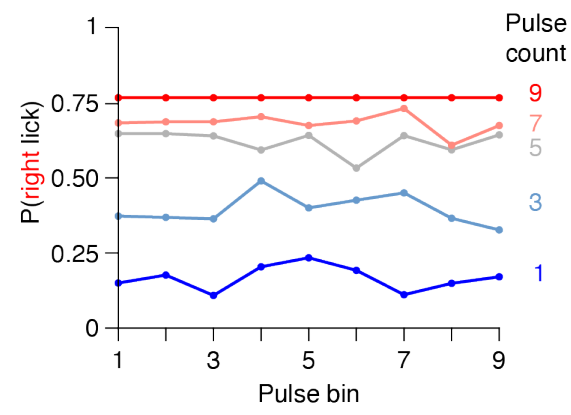

B

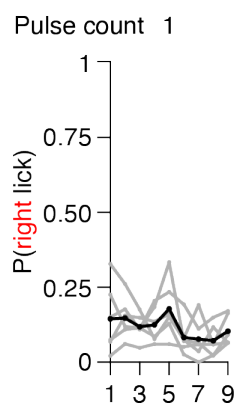

3

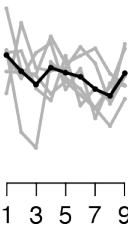

5

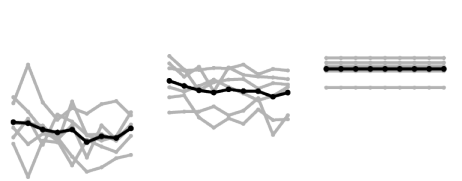

Figure S4, Related to Figure 1. Mice do not use pulse timing to solve the task

(A) Right lick probability in each of the 9 possible pulse time bins for each pulse count in the final discrimination task for one animal.

(B) Same as (A) for all animals. Grey, individual animals; black, mean. 
Pancholi et al., Figure S5

A
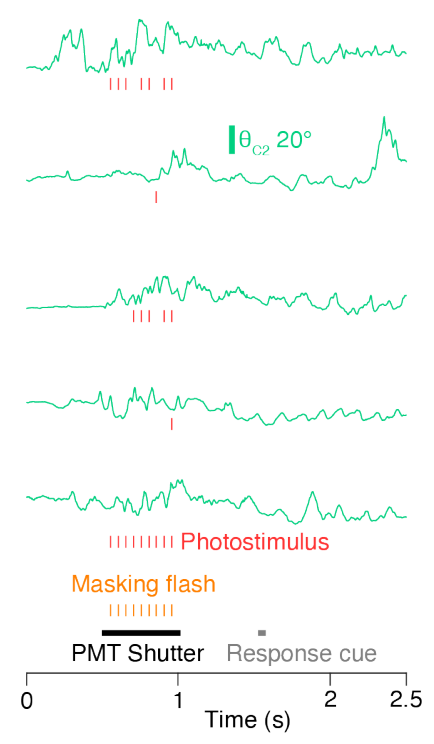

B

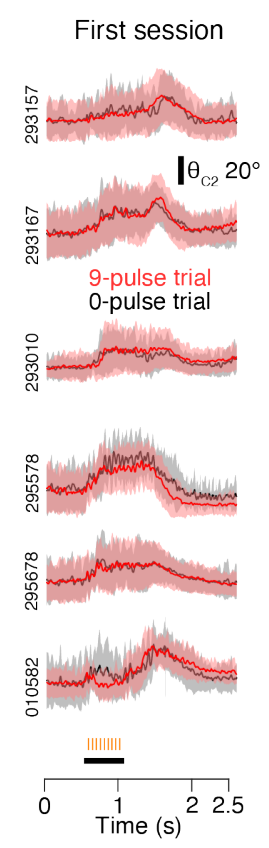

C

Last $9 / 0$ session Full task, early Full task, late
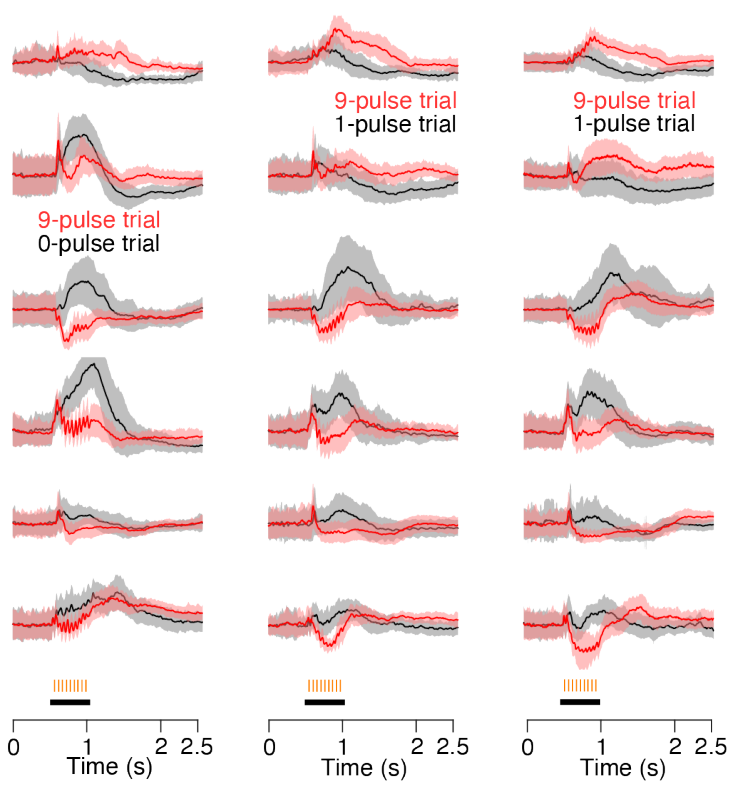

Figure S5, Related to Figure 2. Whisking during the photostimulation epoch

(A) Example trials showing whisker angle (green) in relation to photostimulation (red), the masking flash (orange), the PMT shutter (black), and the response cue (grey).

(B) Average whisking in the first behavioral session for each mouse on 9-pulse (red) and 0 -pulse (black) trials. Shaded region shows SEM across trials, dark line indicates mean. (C) As in (B), but for subsequent days. Left to right: the final session where 0-pulse trials were used; early full task; late full task. 
Pancholi et al., Figure S6

$\mathbf{A}$

Day 1

Day 12

Day 24

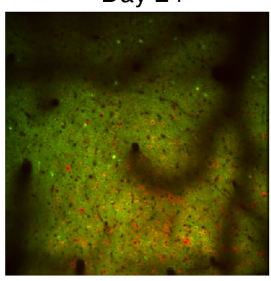

Day 37

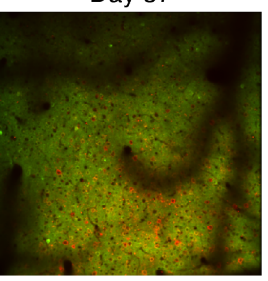

Day 47
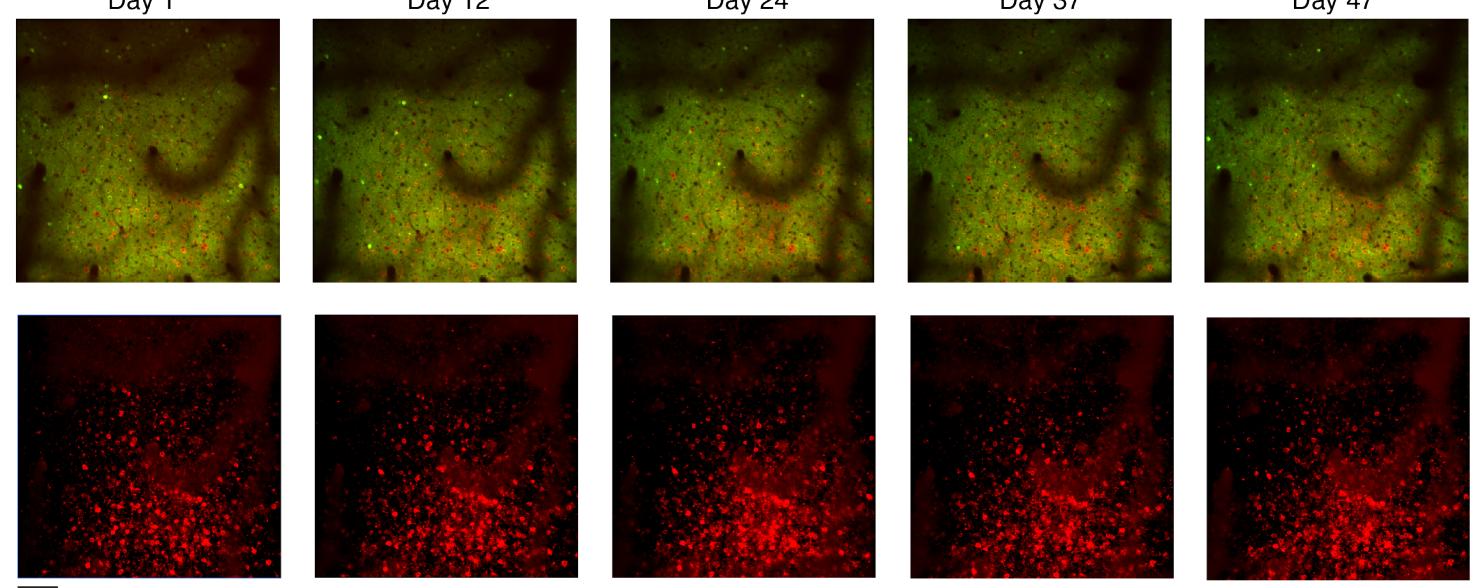

C

B
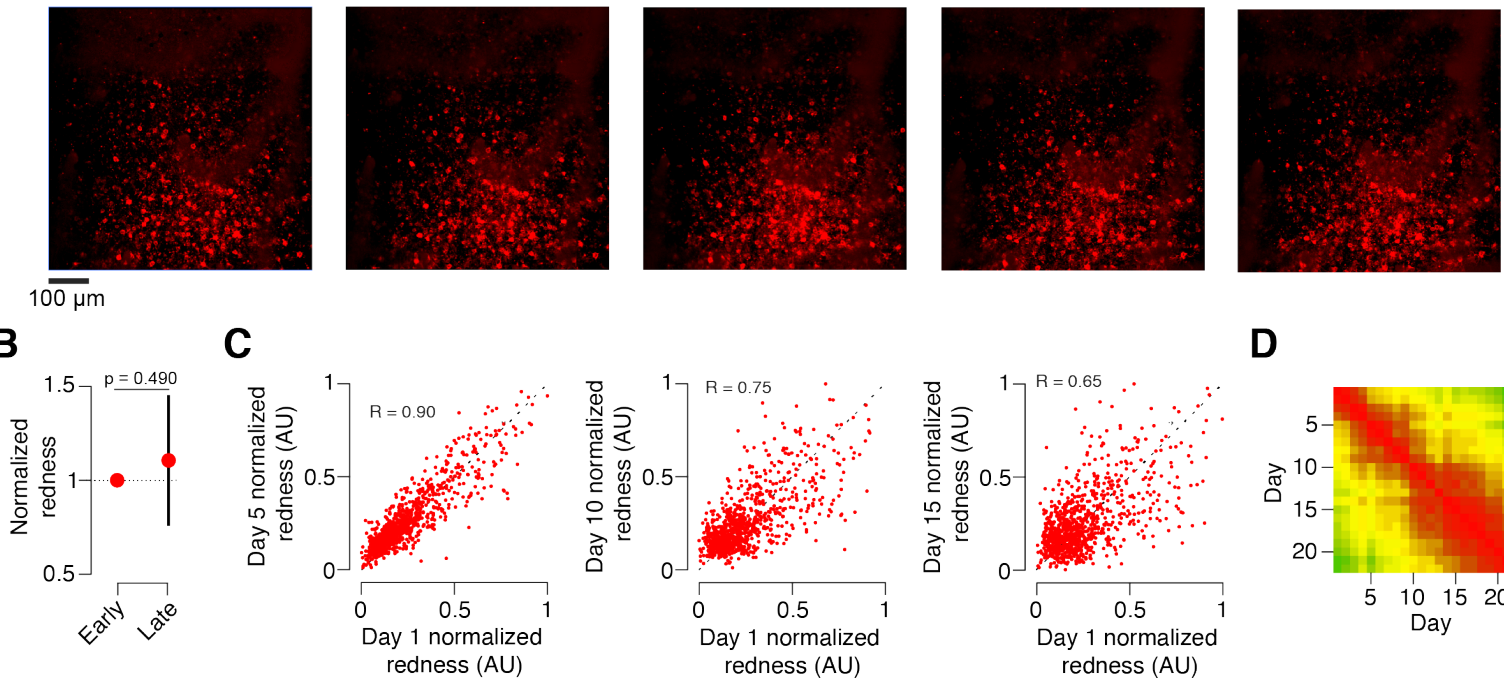

D

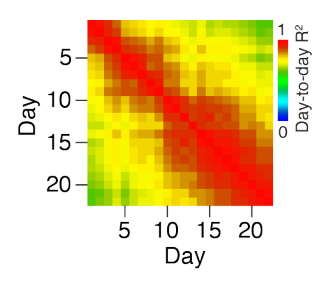

Figure S6, Related to Figure 3. Opsin expression over the course of learning

(A) Example imaging plane across nearly 7 weeks. Top, GCaMP6s (green) and opsin (red) expression; bottom, linearly demixed red channel signal showing opsin expression. (B) Mean redness for the earliest and latest sessions. Error bars, SEM. P-value given for paired t-test comparing early to late sessions ( $n=6$ mice).

(C) Relationship between redness on day 1 and redness on days 5 (left), 10 (center), and 15 (right) for example animal.

(D) Day-to-day correlation of redness across all opsin-expressing neurons averaged across animals ( $n=6$ mice). 
Pancholi et al., Figure S7
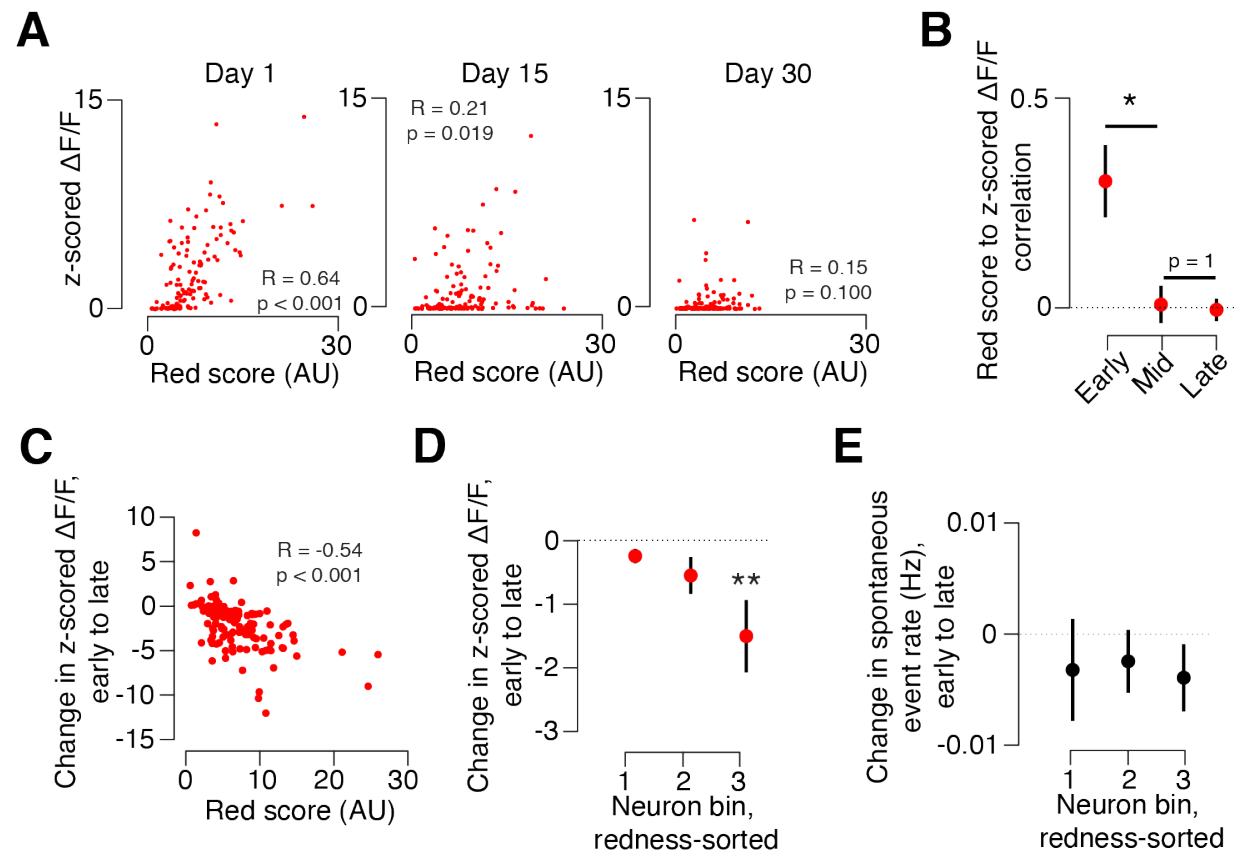

Figure S7, Related to Figure 3. Opsin expression becomes less predictive of neural response.

(A) Example relationship (Pearson correlation) between red score and response amplitude on early, middle, and late days.

(B) Mean correlation between red score and response amplitude for early, middle, and late sessions across mice $(n=6)$. Error bars, SEM.

(C) Relationship between redness on day 1 and the change in response amplitude from early to late sessions.

(D) Change in response amplitude from early to late sessions for 3 bins with equal number of neurons sorted by redness, with bin 3 containing the reddest neurons. Error bars, SEM $\left(n=6\right.$ mice). ${ }^{*} p<0.05,{ }^{* *} p<0.01,{ }^{* * *} p<0.001$, unlabeled $p \geq 0.05$; Bonferroni-adjusted t-test comparing values to distribution with same variance and mean of 0 .

(E) As in (D), but for change in spontaneous event rate $(\mathrm{Hz})$ from early to late sessions. Significance was assessed via Bonferroni-adjusted t-test comparing measured values to a distribution with same variance and mean of $0(n=6$ mice); unlabeled, $p \geq 0.05$. 
Pancholi et al., Figure S8

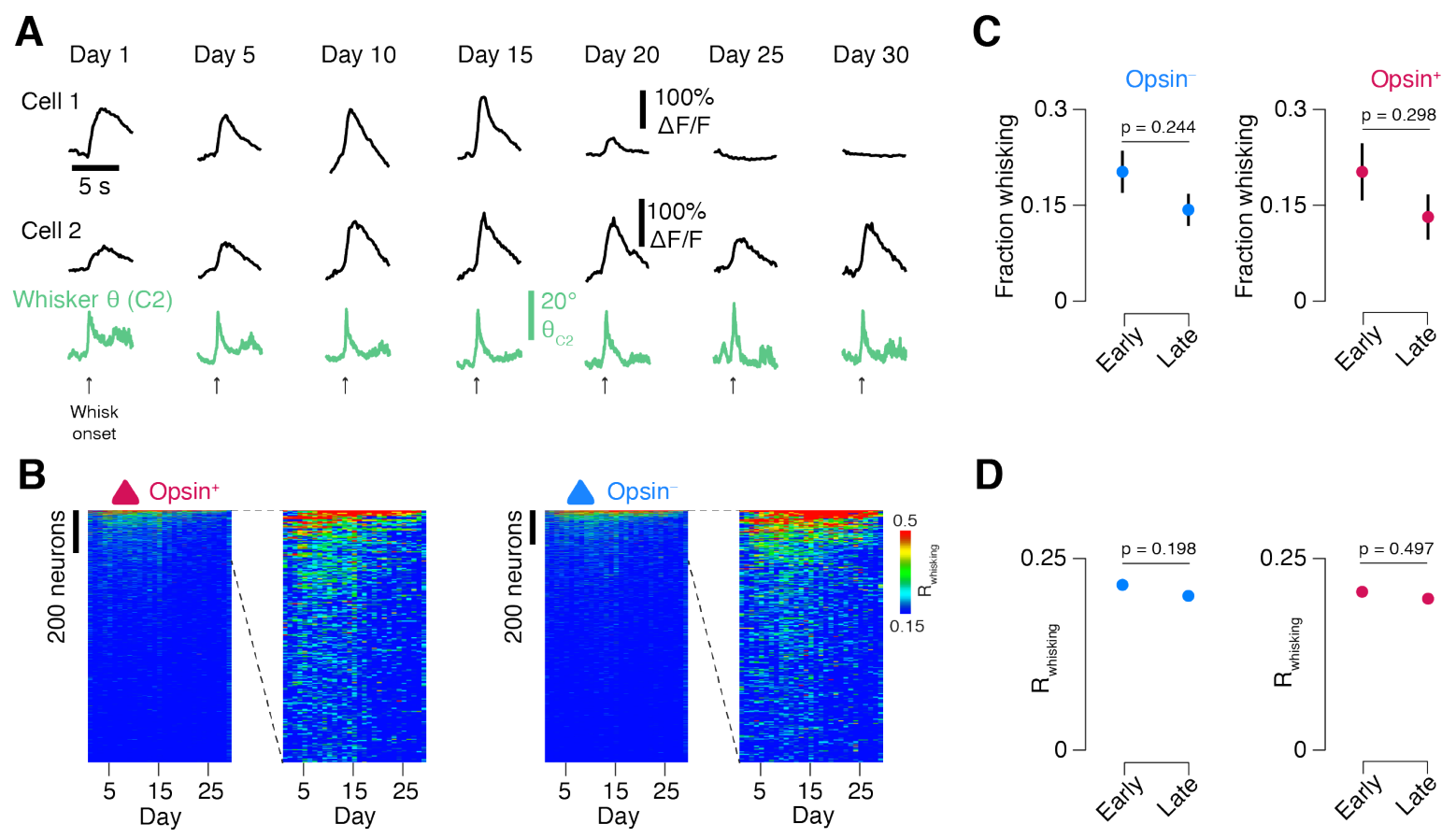

Figure S8, Related to Figure 5. Whisking representations are stable during photostimulation task training.

(A) $\Delta \mathrm{F} / \mathrm{F}$ in two example neurons (top, middle) and whisker angle (bottom, green) across sessions averaged aligned to whisking onset for a given session. Lines show mean across multiple whisks.

(B) Whisking encoding score (Rwhisking; Methods) across sessions for opsin-expressing (left, red) and opsin non-expressing neurons (right, blue) from one example animal.

(C) Fraction of neurons classified as whisking cells on early and late sessions. Left, opsin non-expressing neurons; right: opsin-expressing neurons. Significance indicated for t-test comparing early to late values ( $\mathrm{n}=6$ mice).

(D) Mean encoding score across all whisking cells for early and late sessions. Left, opsin non-expressing neurons; right, opsin-expressing neurons. Significance indicated for t-test comparing early to late values ( $n=6$ mice). 
Pancholi et al., Figure S9

A
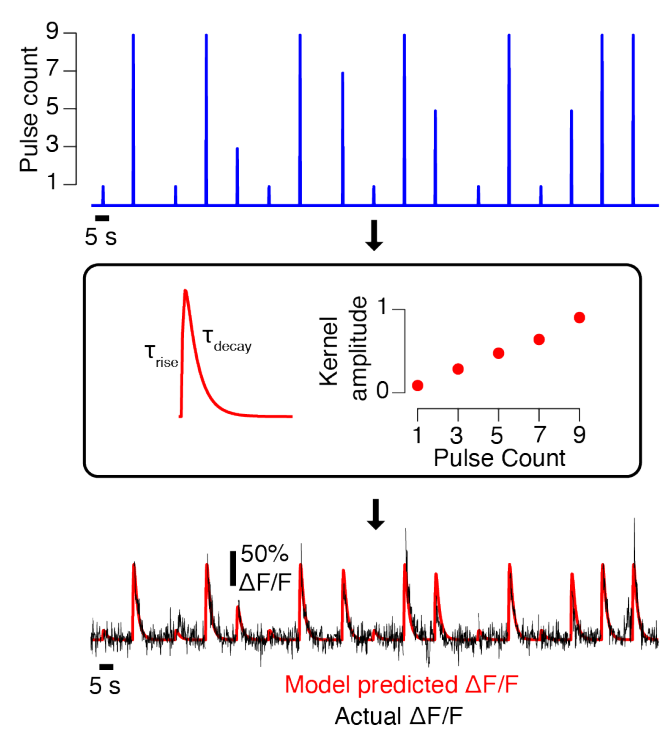

B

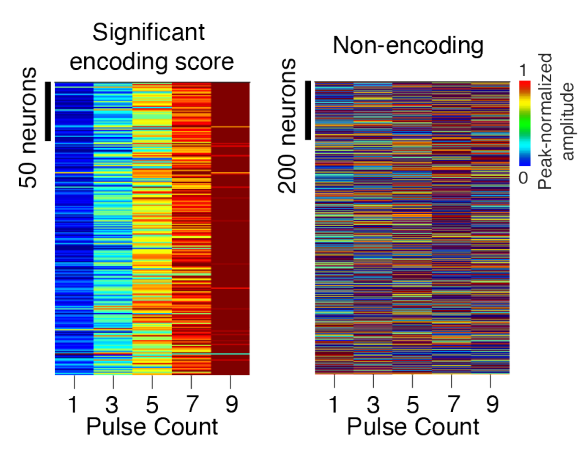

C

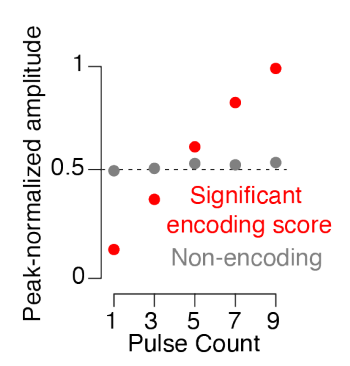

Figure S9, Related to Figure 5. Optical microstimulation encoding model

(A) Optical microstimulation encoding model. Top: the model is provided with the number of stimulation pulses on a given trial and stimulus timing. Middle: the model consists of a temporal kernel with rise and decay time constants, constrained by known GCaMP6s kinetics (Chen et al., 2013). The amplitude kernel allows each pulse count response amplitude to be fit independently of other pulse counts. Bottom: the model generates a predicted $\Delta F / F$ for each neuron.

(B) Peak-normalized amplitudes of example pulse count kernels for one mouse. Left, neurons with a significant model encoding score; right, neurons that do not meet the encoding score significance criteria (Methods).

(C) Mean peak-normalized kernel amplitude across all mice and neurons. Red, neurons with a significant model encoding score; grey, neurons that do not meet encoding criteria. 